\title{
Development of a lightweight camera for high altitude platform systems
}

\author{
Paul C. T. Rees, ${ }^{\mathrm{a}, *}$ Ian P. Baker, ${ }^{\mathrm{b}}$ David A. Thomson, ${ }^{\mathrm{c}}$ Dean Catterall, ${ }^{\mathrm{b}}$ \\ Martin Coleman, ${ }^{d}$ Martyn Jones $\odot,{ }^{a}$ and John B. Mitchell ${ }^{d}$ \\ ${ }^{a}$ Wrexham Glyndŵr University, St. Asaph, United Kingdom \\ ${ }^{\mathrm{b}}$ Gooch and Housego (St. Asaph), St. Asaph, United Kingdom \\ ${ }^{\mathrm{c}}$ Thomson Telescopes Ltd., Great Sutton, United Kingdom \\ ${ }^{\mathrm{d}}$ Glyndŵr Innovations Ltd., OpTIC Centre, St. Asaph, United Kingdom
}

\begin{abstract}
We describe the development of a lightweight, high-resolution surveillance camera for deployment on high altitude platform systems. The instrument is designed to operate at an altitude of $\sim 20 \mathrm{~km}$ and has an expected ground resolution of better than $120 \mathrm{~mm}$ with an appropriate sensor. While designed specifically for imaging at visible wavelengths, it is shown that the design is capable of diffraction-limited imaging at NIR and SWIR wavelengths up to $2.5 \mu \mathrm{m}$. We have combined a range of materials from aluminum and titanium alloys through to carbon fiberreinforced plastic to produce an instrument with structural components that match the thermal expansion of the optical glasses used. The use of these materials has resulted in an instrument that weighs $<2 \mathrm{~kg}$, including a sensor package, and is designed to weigh $<3 \mathrm{~kg}$ once integrated with an enclosure and actuated gimbal. The successful testing of two prototype systems is described, including several design outcomes from the program intended for implementation in advance of flight trials. (c) The Authors. Published by SPIE under a Creative Commons Attribution 4.0 Unported License. Distribution or reproduction of this work in whole or in part requires full attribution of the original publication, including its DOI. [DOI: 10.1117/1.OE.59.10.105104]
\end{abstract}

Keywords: camera; reconnaissance; lightweight; high altitude.

Paper 20200850 received Jul. 13, 2020; accepted for publication Sep. 18, 2020; published online Oct. 20, 2020.

\section{Introduction}

There is an emerging market for persistent airborne surveillance and high-bandwidth freespace optical communications using high altitude platform systems (HAPS). In 2015, Glyndŵr Innovations Limited successfully bid for funding from the UK Defence Science and Technology Laboratory as part of its "Research and Development into Persistent Surveillance - Challenge 1" to develop a lightweight imaging system targeted at HAPS deployment. Following successful delivery of phase 1, a second phase of development was funded later in 2016 to design and manufacture a flight-capable prototype system.

This paper describes the development of an imaging optical system (henceforth "camera system") for HAPS deployment, designed to meet the requirements set by a high-resolution imaging application (panchromatic, multispectral, or hyperspectral) or to act as the front-end to a LIDAR instrument. These application areas were chosen to represent the range of optical systems expected to be deployed on HAPS, including the requirements for free-space optical communications. ${ }^{1,2}$ Applications such as these require challenging optical performance specifications as well as the engineering requirements specific to HAPS deployment. ${ }^{3}$ In addition, most development activity for this technology is focused on the development of high altitude long endurance (HALE) operations. For this mode of operation, a demanding set of SWaP (space, weight, and power) requirements is also established to maximize mission durations.

The optical system described herein is a diffraction-limited modified Cassegrain telescope of $150-\mathrm{mm}$ aperture operating throughout the visible spectrum. The performance requirement is to attain a nominal ground resolution of $100 \mathrm{~mm}$ from stratospheric altitudes. While this demonstrator

*Address all correspondence to Paul C. T. Rees, p.rees@glyndwr.ac.uk 
system is limited to imaging applications at visible wavelengths, the design is intended to act as a prototype for the fore optics for a LIDAR system with only minor modification. The end use of a system of this design is surveillance and/or free-space optical communications.

\section{Requirements}

The challenge for the design of optical systems operating on HAPS comes with the combination of expected operating environment and the payload SWaP requirements of the platform. These requirements are discussed in what follows.

\subsection{Optical Requirements}

The required optical performance of the system is summarized in Table 1. Notably, the swath, or projected field of view (FOV), was not defined. For this exercise, the swath was to be "as large as the design will reasonably allow."

\subsection{Environmental Requirements}

The environmental requirements are those of an instrument operating with only nominal protection from the external atmospheric conditions at an operating altitude of $\sim 21.3 \mathrm{~km}(70,000 \mathrm{ft})$. These conditions can be summarized using the International Standard Atmosphere; ${ }^{4}$ i.e., an ambient temperature of $-55^{\circ} \mathrm{C}$ and an atmospheric pressure of 45 mbar. The relative humidity is expected to be close to zero at operating altitude, but during ascent and descent the relative humidity can be expected to reach $100 \%$ for periods. In addition, heating from solar radiation can vary between 700 and $1300 \mathrm{Wm}^{-2}$ during the daytime to zero during the night-time.

From the above, an operating temperature range of $-40^{\circ} \mathrm{C}$ to $+30^{\circ} \mathrm{C}$ was defined. This temperature range includes laboratory and ground set-up temperatures, those experienced by the instrument during ascent and descent, and operation at altitude within a "simple" aerodynamic enclosure.

A survival temperature range was defined from $-80^{\circ} \mathrm{C}$ to $-40^{\circ} \mathrm{C}$ and from $+30^{\circ} \mathrm{C}$ to $+40^{\circ} \mathrm{C}$. Over these temperature ranges, the instrument is expected to survive and to recover its specified optical performance once it returns to its operating temperature range.

These environmental parameters are summarized in Table 2. Note that the survival temperature range is intended to encompass the night-time temperature, but that night-time operation is not envisaged.

\subsection{SWaP Requirements}

The SWaP requirements for these platforms vary with type of platform. For fixed-wing aircraft (for example, the Airbus Zephyr platform), a documented payload weight of up to $5 \mathrm{~kg}$ is defined, ${ }^{5}$ with a maximum payload power consumption of $250 \mathrm{~W}$ and an instrument pod volume of between 15,000 and $24000 \mathrm{~cm}^{3}$, depending upon the geometry of the payload. Additional

Table 1 Optical specification characteristics for the proposed optical system.

\begin{tabular}{lc}
\hline \hline Parameter & Assumed value \\
\hline Aperture & $150 \mathrm{~mm}$ \\
Operating wavelength range & 450 to $750 \mathrm{~nm}$ \\
Reference wavelength & $550 \mathrm{~nm}$ \\
Ground resolution & $100 \mathrm{~mm}$ \\
Swath & To be defined \\
\hline \hline
\end{tabular}


Rees et al.: Development of a lightweight camera for high altitude platform systems

Table 2 Specified environmental requirements adopted for the instrument.

\begin{tabular}{lr}
\hline \hline Parameter & Assumed value \\
\hline Operating temperature range & $-40^{\circ} \mathrm{C}$ to $+30^{\circ} \mathrm{C}$ \\
Survival temperature range & $-80^{\circ} \mathrm{C}$ to $-40^{\circ} \mathrm{C}$ \\
& $+30^{\circ} \mathrm{C}$ to $+40^{\circ} \mathrm{C}$ \\
Operating atmospheric pressure range & 40 to $1000 \mathrm{mbar}$ \\
Solar heating during operation & 700 to $1300 \mathrm{Wm}^{-2}$ \\
\hline \hline
\end{tabular}

Table 3 Specified SWaP requirements adopted for the instrument.

\begin{tabular}{|c|c|c|}
\hline Parameter & Assumed value & Comments \\
\hline $\begin{array}{l}\text { Maximum nose } \\
\text { pod volume }\end{array}$ & $24,000,000 \mathrm{~mm}^{3}$ & $\begin{array}{l}\text { The nose pod is assumed to be custom-made to suit } \\
\text { the dimensions, weight and functionality of the payload }\end{array}$ \\
\hline $\begin{array}{l}\text { Instrument space } \\
\text { envelope }\end{array}$ & $\begin{array}{l}150 \mathrm{~mm} \times 150 \mathrm{~mm} \times \\
400 \mathrm{~mm}\end{array}$ & The target volume defined for the imaging instrument \\
\hline Payload mass & $<3 \mathrm{~kg}$ & $\begin{array}{l}\text { Payloads of }>3 \mathrm{~kg} \text { but }<5 \mathrm{~kg} \text { are possible, but will have } \\
\text { a detrimental effect on the platform mission capability }\end{array}$ \\
\hline Electrical power & $<250 \mathrm{~W}$ & Assumed to be peak power consumption \\
\hline
\end{tabular}

space for payload storage is available within the wing sections of the aircraft. Inflatable HAPS vehicles (for example, the Thales Alena Stratobus), can allow payloads of $250 \mathrm{~kg}$ or greater and up to $5-\mathrm{kW}$ payload power. For the instrument described herein, the more demanding SWaP requirements for a fixed-wing HAPS were adopted.

The defined SWaP requirements are presented in Table 3.

The dimensions of the instrument space envelope were a self-imposed goal. They were imposed to ensure a provision was made for other instrumentation within the pod with the camera system acting as front-end optics.

\subsection{Other Requirements}

Two further requirements remained unspecified throughout the development process but need mention:

(1) A maximum shock loading for the system, representative of landing.

(2) A vibration spectrum for the platform, representative of the vibration expected while flying at operational altitudes.

Neither of these quantities was freely available during the development of the optical system. Because of this, they were estimated (Table 4) from published studies of vibration modes of composite aircraft. ${ }^{6,7}$

Table 4 Specified frequency-related loading requirements adopted for the instrument.

\begin{tabular}{lcl}
\hline \hline Parameter & Assumed value & \multicolumn{1}{c}{ Comments } \\
\hline Maximum shock loading & $2.5 \mathrm{~g}$ & In any one direction \\
Safety factor & 2.0 & To be applied to maximum shock loading \\
Maximum modal frequency of the platform & $50 \mathrm{~Hz}$ & Assumed \\
\hline \hline
\end{tabular}




\section{Optical Design}

\subsection{Detector Choice}

At an altitude of $21.3 \mathrm{~km}$, the target resolution of $100 \mathrm{~mm}$ subtends an angle of $4.7 \mu \mathrm{rad}$, or $0.97 \mathrm{arcsec}$. At a reference wavelength of $550 \mathrm{~nm}$, a $150-\mathrm{mm}$ circular aperture has an Airy disc radius of $4.5 \mu \mathrm{rad}$ or $0.92 \mathrm{arcsec}$. This suggests that resolving $100 \mathrm{~mm}$ from the stratosphere with an instrument of $150 \mathrm{~mm}$ aperture is feasible without postprocessing of image data, although close to the physical diffraction limit. To achieve this resolution, the focal plane must be adequately sampled. For modern sensors of 3 to $6 \mu \mathrm{m}$ pixel pitch, an effective focal length (EFL) in the range 700 to $1500 \mathrm{~mm}$ is required to satisfy the Nyquist sampling criterion.

After a review of suitable sensors, a packaged industrial vision camera using a Sony EXview HAD II ICZ814 CCD sensor was chosen as a demonstrator sensor. The sensor chosen is monochromatic (from 400 to $1000 \mathrm{~nm}$ wavelength), has a square pixel pitch of side $3.69 \mu \mathrm{m}$, and a detector area of $3376 \times 2704$ pixels. These pixel dimensions indicate an EFL of $>850 \mathrm{~mm}$ is needed to achieve the required ground resolution (i.e., a focal ratio $>f / 5.5$ ).

\subsection{Telescope Design}

A review of possible optical designs against the SWaP requirements indicated that an on-axis reflective telescope design is most likely to meet the design volume and weight requirements. Our review indicated that off-axis designs with an aperture of $150 \mathrm{~mm}$, e.g., three-mirror anastigmats $\left(\operatorname{Korsch}^{8}\right)$, are at a disadvantage because of the limited design volume available. Also, Cassegrain designs with a full-aperture transmissive corrector (e.g., Maksutov ${ }^{8}$ or $\mathrm{Schmidt}^{8}$ designs) are at a weight disadvantage in this application. Designs with a single full-aperture optic such as the Classical Cassegrain ${ }^{8}$ or Ritchey-Chrétien ${ }^{8}$ designs are favored. Of the Cassegrain designs, the Ritchey-Chrétien design was selected. This design, modified with a Wynne ${ }^{9}$-style refractive field curvature corrector, can deliver a well-corrected FOV of suitable size.

An EFL of $900 \mathrm{~mm}$ (i.e., a focal ratio $f / 6$ ) was selected for the reflective Cassegrain telescope. A primary mirror focal length of $300 \mathrm{~mm}$ (focal ratio $f / 2$ ) was selected as a near optimum balance between the compactness of the telescope design and the predicted alignment sensitivity. The design of the Wynne corrector modifies the EFL of the telescope system slightly to give an EFL of $\sim 914 \mathrm{~mm}$.

The optical design was modeled using Zemax OpticStudio optical design software. The optical design is shown in Fig. 1. The large separation between the primary mirror vertex and the focal plane in this design is to accommodate the support for the mirror, a focusing assembly, and an interface between the telescope structure and a gimbal for pointing.

The glass selected for the corrector lens elements was Schott N-BK7. One corrector lens is planoconvex in form and the other planoconcave. This design provides a well-corrected FOV of $>1.2 \mathrm{deg}$ at visible wavelengths (i.e., from 450 to $750 \mathrm{~nm}$ ). Image quality degrades rapidly for

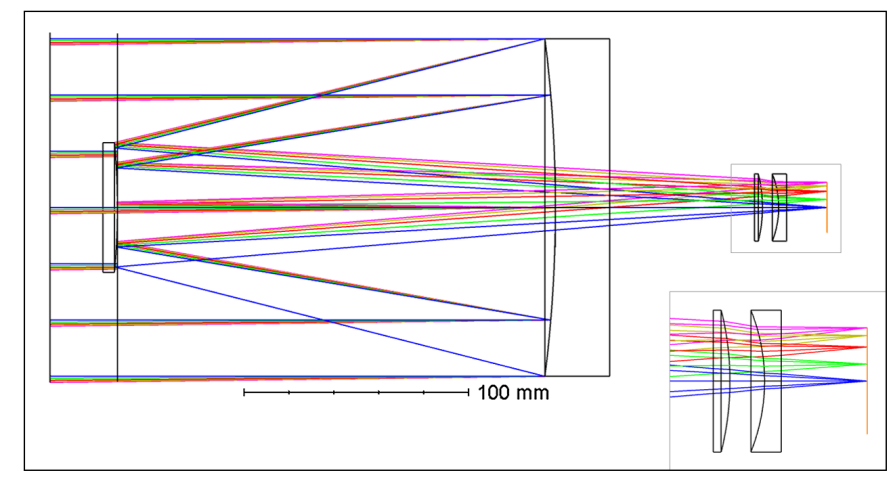

Fig. 1 The optical layout of the telescope design, incoming beam to the left, focal plane on the right. Colored rays represent different field points, from field center to field edge. 
fields $>1.4$ deg in diameter. A three-element corrector is also feasible and can produce a wellcorrected FOV of 1.9 deg for the same Cassegrain telescope design.

A representative spot diagram is presented in Fig. 2. It is clear from Fig. 2 that the design is capable of diffraction-limited performance from $450 \mathrm{~nm}$ (blue) through to $2.5 \mu \mathrm{m}$ (SWIR) wavelengths. This performance is valuable for use in simultaneous imaging in the visible and NIR/SWIR or in LIDAR applications.

Notwithstanding wavefront degradation from optical fabrication and integration tolerances, the ground resolution of the telescope system operating at an altitude of $21.3 \mathrm{~km}$ is the product of the optical resolution delivered to the camera sensor and the sampling of this image by the sensor. The ability to resolve detail at the ground-based target will also depend upon the contrast apparent at the target and the signal/noise ratio of the image data.

The modeled modulation transfer function (MTF) of the system is shown in Fig. 3. Using the pixel pitch for the chosen sensor, Sony EXview HAD II ICX814, the combined MTF of the optical system and the camera sensor can be approximated [Fig. 3(b)]. In Fig. 3(b), the modulation data at the target are calculated by approximating the CCD pixel as a rectangle function. While this approximation is idealized, it modulates the optical MTF sufficiently realistically to illustrate the effects of sensor pixel size on system resolution.

The system resolution will also be affected by noise in the image data. In this context, we define "noise" as any source of infidelity in the expected mapping of the object onto the recorded image. Noise can therefore originate both outside of and within the camera system, including detector noise. By this definition, noise may be random, pseudorandom, or nonrandom. If this noise contribution is approximated by a "white" frequency distribution and is assumed to be pseudorandom in nature, then the effect of a noise equivalent modulation (NEM) upon resolution can be estimated. In Fig. 3(b), the NEM is estimated to be 0.05 . This is intended to represent the lowest useful modulation for a quantitative application.

The intersection of the NEM and the predicted system modulation represents the expected maximum resolution of the system. Figure 3(b) shows that a ground resolution of $100 \mathrm{~mm}$ is unlikely with this instrument when using a sensor of 3.69- $\mu \mathrm{m}$ pixel size. A maximum pixel size of $3.0 \mu \mathrm{m}$ is necessary to match the performance of the optical instrument.

In summary, a practical optical design has been developed. The full projected aperture of the focal plane is $520 \mathrm{~m}$ at the target, nadir pointing, corresponding to an optical FOV of $1.4 \mathrm{deg}$, and a focal plane of $22.3 \mathrm{~mm}$ diameter. In combination with the selected sensor, the instrument is expected to deliver a ground resolution of $\sim 113 \mathrm{~mm}$ and offer a swath of $\sim 290 \times 233 \mathrm{~m}^{2}$.

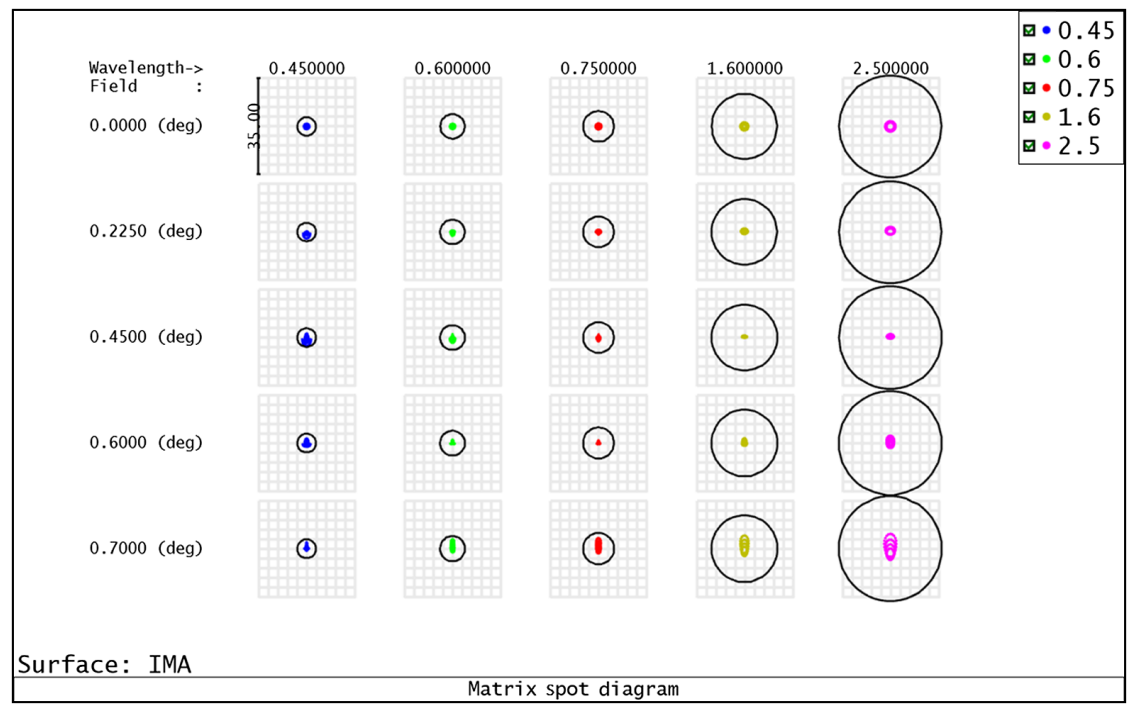

Fig. 2 The matrix spot diagram for the optical system, showing the optical performance of the design from $450 \mathrm{~nm}$ to $2.5 \mu \mathrm{m}$ wavelength. Solid circles represent the Airy disc. 


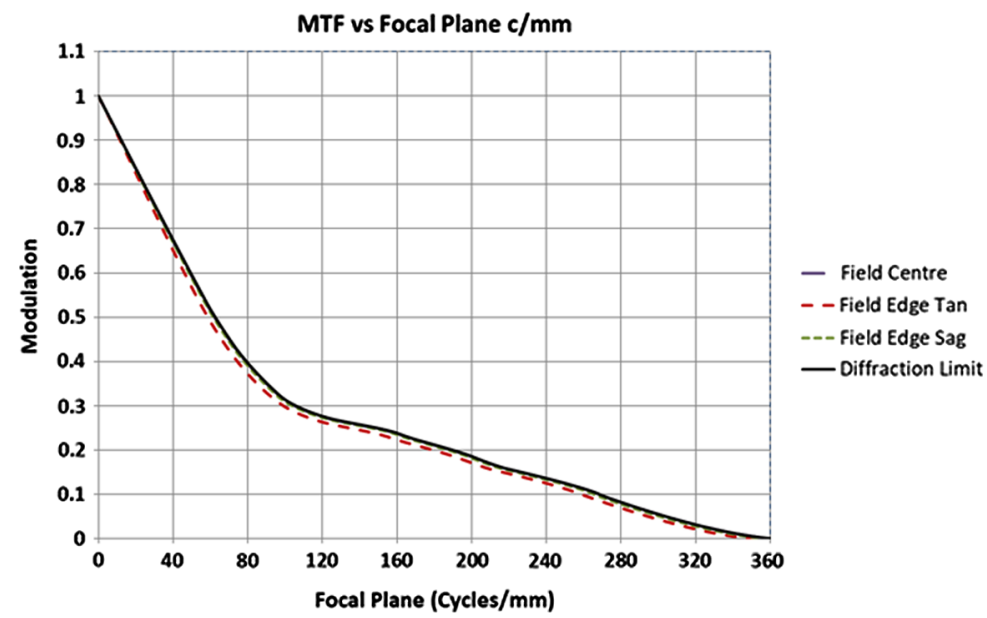

(a)

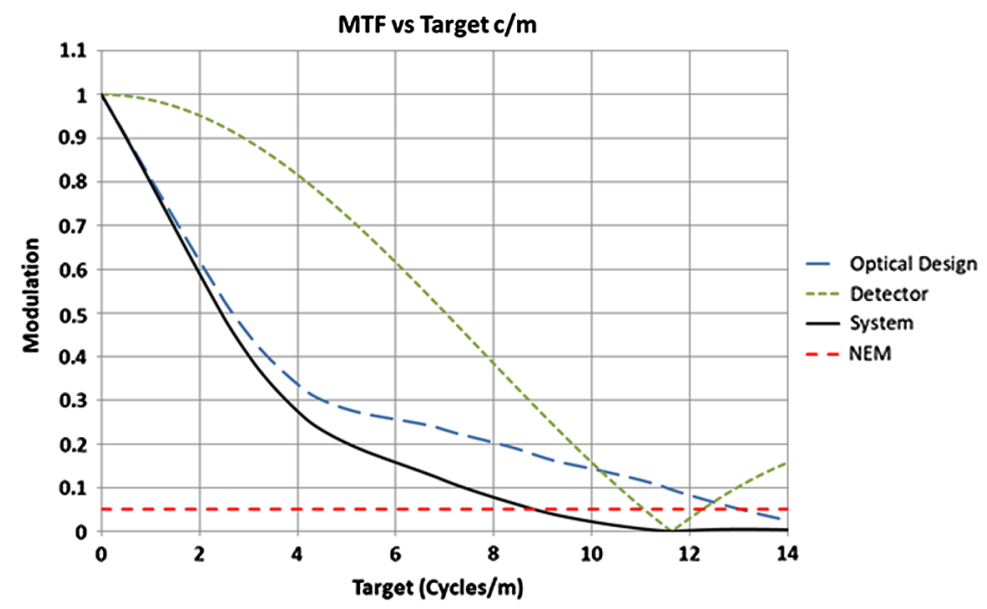

(b)

Fig. 3 (a) The predicted MTF of the system, showing the image resolution performance of the system shown in Fig. 1. (b) The predicted MTF in target units, including the effects of sensor pixel sampling.

\section{Optomechanical Design}

Several key factors affect the optomechanical design of the camera system:

- The mass of the primary mirror.

- The mounting of each of the optics to the telescope structure.

- The alignment of each of the optics within the structure.

None of these factors are uncommon, but the environmental and SWaP requirements place the optomechanical design within a challenging context.

All optical components used N-BK7 as a substrate material, including the mirrors. This choice was intended to match the coefficient of thermal expansion (CTE) of the materials used in the structure of the instrument; while using diverse materials (see Sec. 5), initial analysis indicated an expected average structural CTE in the range $7 \times 10^{-6} /{ }^{\circ} \mathrm{C}$ to $10 \times 10^{-6} /{ }^{\circ} \mathrm{C}$.

The rear of the primary mirror was light-weighted (Fig. 4) to optimize its mass. The lightweighting removed $\sim 63 \%$ of the monolithic mass of the primary mirror.

The mounting arrangement of the primary mirror uses the central bore of the mirror, mounted to an aluminum alloy (7075) cylinder with an elastomer interface (Fig. 5). No bonding is used in this mounting arrangement. Fine alignment of the primary mirror is via three locking fine screw adjusters that bear on the aluminum cell. 


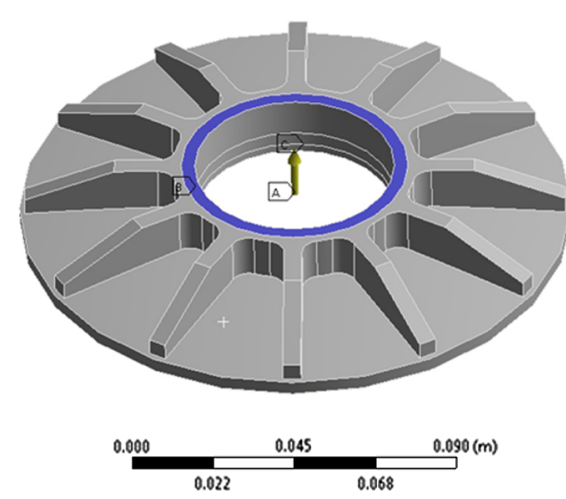

(a)

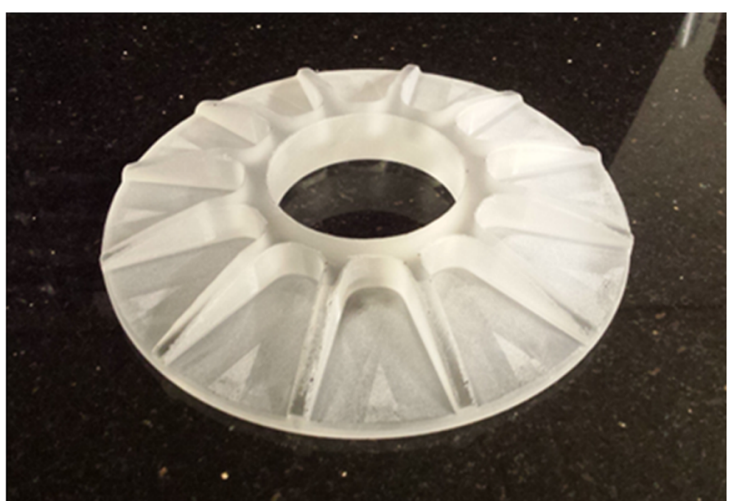

(b)

Fig. 4 (a) Model of the primary mirror light-weighting and (b) a light-weighted primary mirror during fabrication.

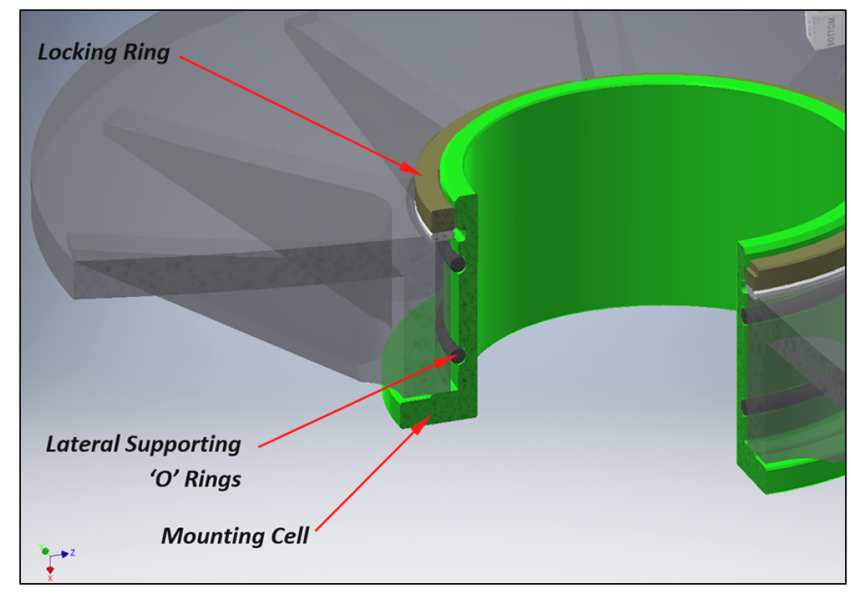

Fig. 5 Mounting arrangement for the primary mirror. The cell is machined aluminum alloy (7075).

The light-weighting pattern was developed to deliver good performance against gravitationally driven deformation (Fig. 6): finite element analysis (FEA) was used to predict a maximum gravitationally induced deformation of the optical surface of $\sim 9$-nm peak-to-valley (PV) for this mirror.

Throughout this design program, the FE model was constructed using Autodesk Inventor mechanical design software, and ANSYS FEA software was used to model both deformation behavior and thermal behavior. For the FEA, the model for the primary mirror was meshed with tetrahedron elements (Fig. 7), and the material properties applied were those defined for N-BK7. The model was constrained on the two surfaces that are in direct contact with the mounting cell: the central bore and the rear plano surface adjacent to the central bore. Gravity was applied as an acceleration simulating the optic at each model orientation, and the mesh of the model was refined until the convergence was $<5 \%$.

The secondary mirror was fabricated as a thin mirror of $6.5-\mathrm{mm}$ center thickness with a plano back. The mounting arrangement for the secondary mirror uses a machined titanium alloy (grade 5) support plate. The mirror back is bonded to this support plate using a structural epoxy resin. The support plate, or cell, is mounted in a cylindrical secondary mirror baffle (Fig. 8). Tilt alignment adjustment of the mirror is via three locking fine screw adjusters that bear on the titanium cell.

Titanium alloy is used for the secondary mirror cell because of the small center thickness of the mirror: the titanium alloy has a good CTE match with that of Schott N-BK7 glass and so reduces the risk of thermally induced deformation of the mirror. 


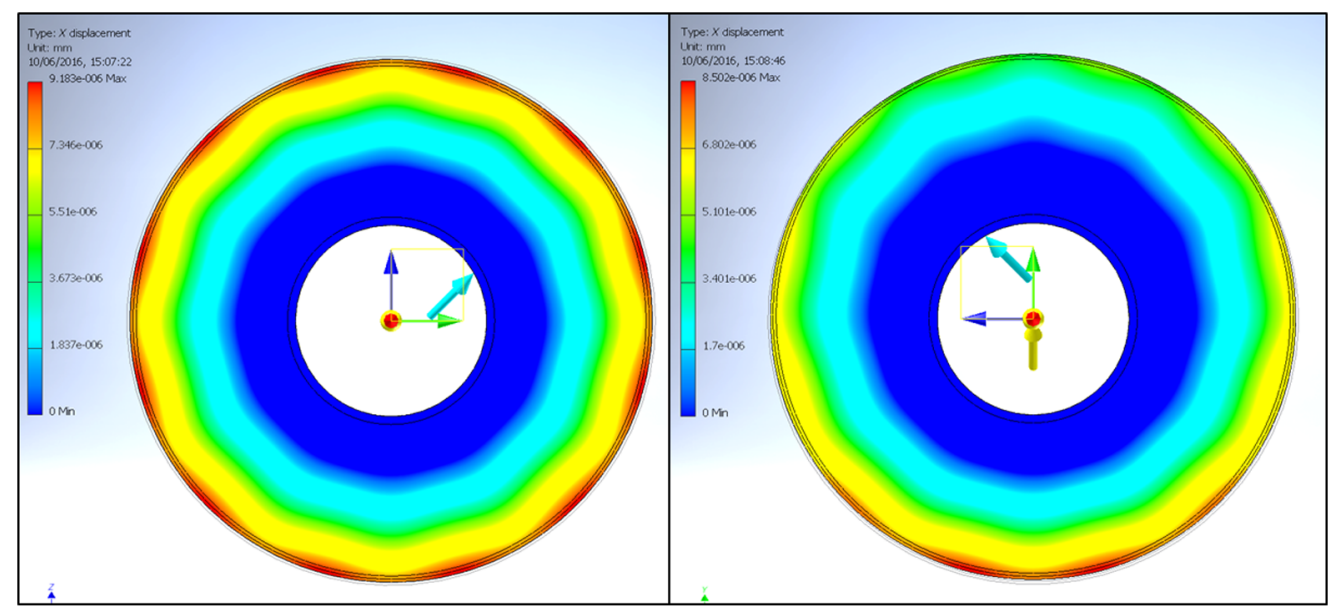

(a)

(b)

Fig. 6 FEA results for gravitationally induced deformation of the light-weighted primary mirror: (a) results for nadir pointing and (b) results for 40 deg to nadir.

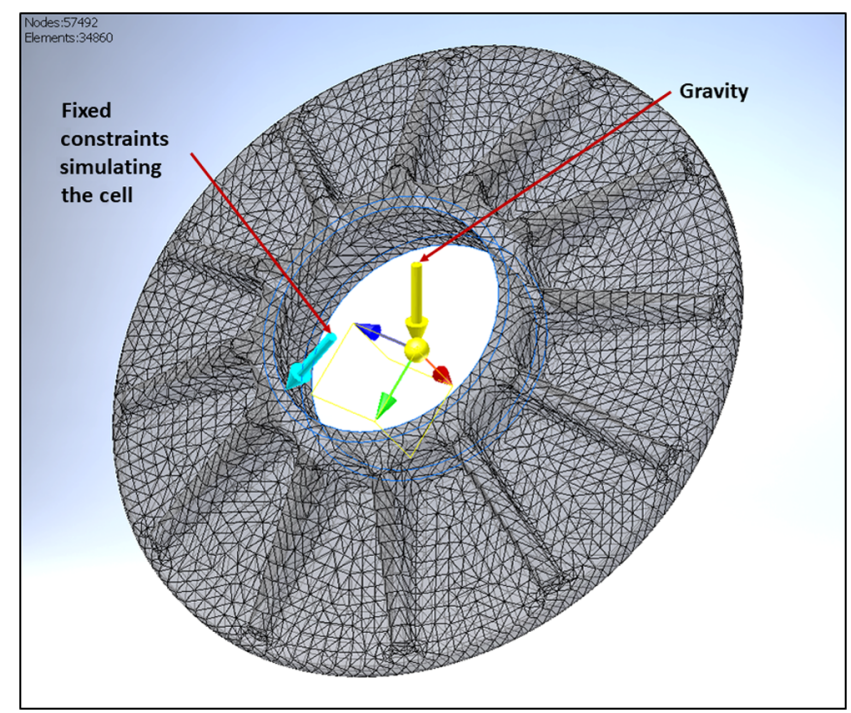

Fig. 7 Finite element mesh used for the analysis of gravitationally induced deformation of the primary mirror.

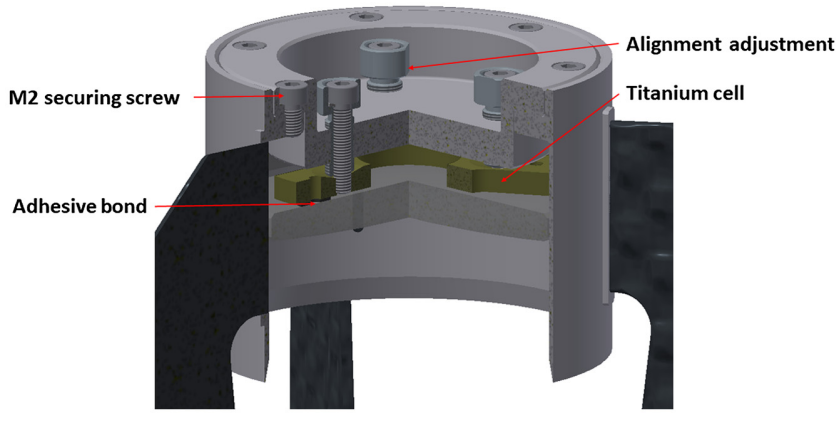

Fig. 8 Mounting arrangement for the secondary mirror. The cell is machined titanium alloy (grade 5). 


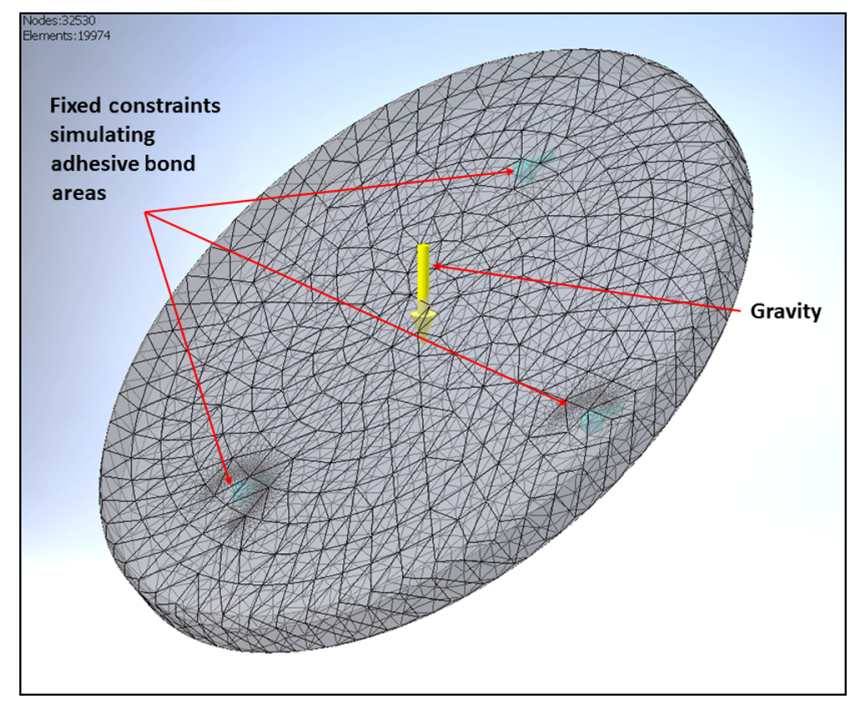

Fig. 9 Finite element mesh used for the analysis of gravitationally induced deformation of the secondary mirror.

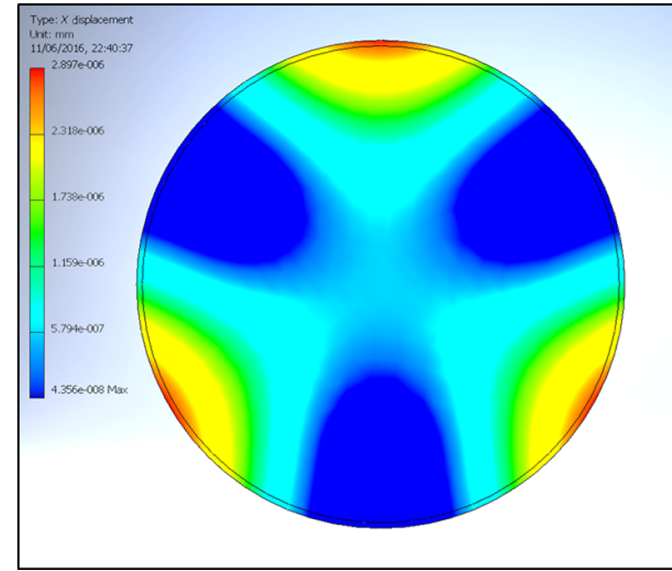

(a)

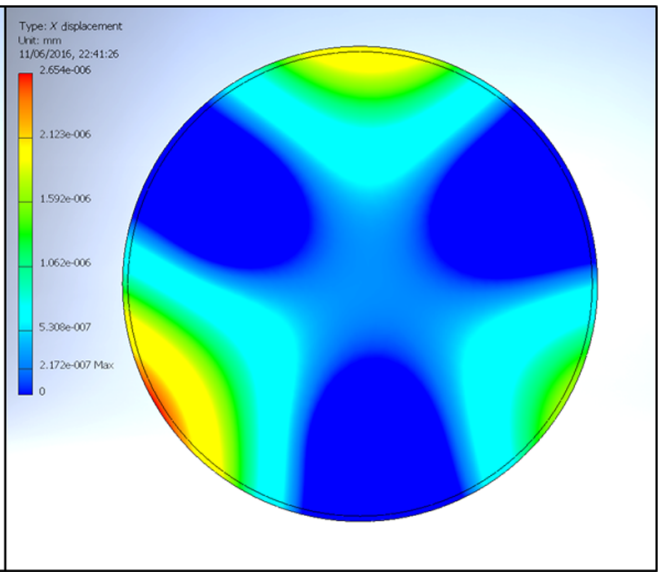

(b)

Fig. 10 FEA results for gravitationally induced deformation of the secondary mirror: (a) results for nadir pointing and (b) results for 40 deg to nadir.

For the FEA, the model of the secondary mirror was meshed with tetrahedron elements and used the material properties defined for N-BK7 (Fig. 9). The model was constrained on the three 7-mm-diameter adhesive bond areas that attach the secondary mirror to its cell assembly. Gravity was applied as an acceleration simulating the optic at each model orientation, and the mesh of the model was refined until the convergence was $<5 \%$.

The FEA results are presented for the secondary mirror in Fig. 10: the analysis predicts a maximum gravitationally induced deformation of the optical surface of $<3-\mathrm{nm}$ PV. Note that the trefoil-shaped deformation of the secondary mirror is attributed to its mounting arrangement.

\section{Mechanical Design}

The factors that affect the mechanical design of the structure of the camera system are the expected thermal behavior of the system, and the effects of gravity vector and vibration. Several different manufacturing processes and materials were considered during the design process. These included: 
(1) A titanium alloy only structure, manufactured by direct metal laser sintering. This offers a good match for the CTE of the optical glass, ensuring a near athermal design. However, a process must be developed to treat parts that will interface to the optics, specifically to avoid hysteretic thermal behavior. Suppliers of sintered titanium alloy also raised concerns regarding possible deformation of the vanes during the build process and could not guarantee manufacture of the structure.

(2) A structure mostly fabricated from aluminum alloy, manufactured using precision machining production techniques. This design is arguably the easiest to manufacture but would not provide a design that behaves athermally because of the mismatch in CTE between structure and optics. As a result, an actuated focus mechanism is a requirement for this design approach and may require some level of real-time focus control during operation.

(3) A structural design using a combination of materials, including: carbon fiber-reinforced plastic (CFRP), titanium alloys, aluminum alloys, and plastics. This design solution offers the opportunity to "tune" the structure design to ensure its thermal expansion closely matches that of the optical components. However, because of the diversity of materials used and the specified operating temperature range, this approach requires the careful use of structural adhesives.

Option (3) was chosen for the material selection in the design of the structure. A numerical model was used to determine the most suitable materials for each structural component. Consideration was given to the manufacturability of the parts as well as their impact on the thermal expansion of the structure. Figure 11 shows the final design of the instrument, as modeled in Autodesk Inventor.

The design of the tube assembly is driven by the need to baffle the optical system effectively and to minimize mass. The design provides a good stiffness to weight ratio. It is suited to applications that require a low mass solution, and where an external housing provides sufficient protection from sources of transient forces (such as wind) and from excessive amounts of stray light.

The three vane components are fabricated from commercial off-the-shelf (COTS) CFRP to provide a high degree of axial stiffness. Two CFRP products were investigated: a bespoke CFRP manufactured at the Advanced Composite Training and Development Centre, Wrexham Glyndŵr University, and a COTS product. The testing program indicated that the bespoke CFRP had a lower CTE but that the COTS CFRP recorded significantly higher stiffness. Each vane is cut by water jet from a 2-mm thickness CFRP sheet.

The primary mirror and secondary mirror baffles have been designed to minimize stray light based upon a geometric model.

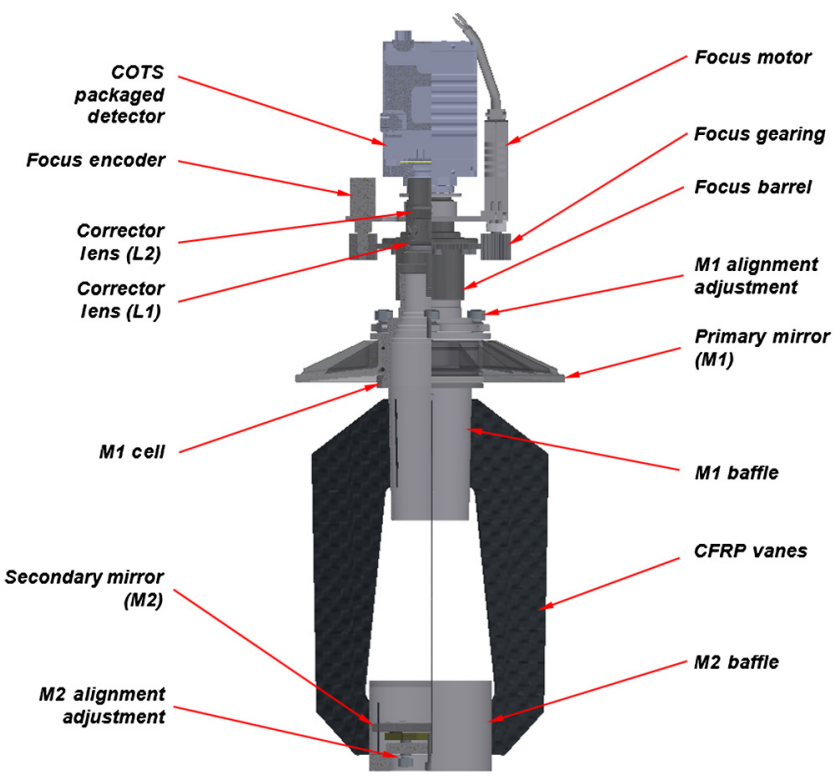

Fig. 11 A schematic view of the design of the camera system, showing its main components. 
The structure at the rear of the primary mirror is tubular to maximize its stiffness. It provides an interface to the sensor package, it allows the incorporation of a focus mechanism, and it provides an interface with a gimbal. Each of the tubular sections is fabricated from machined titanium alloy and assembled using suitably sized fine pitch screw threads. The developed design removes the need for a wide yolk structure, allowing the tube to be supported at the rear of the primary mirror with only a small out-of-balance torque.

As has been discussed, a COTS machine vision camera sensor was chosen for the technical demonstrator system to provide images for system testing and evaluation. While not intended as a choice for operational use, this camera sensor provides a representative payload mass and power dissipation.

FEA of the static deflections resulting from variation in gravity vector indicate decentration between primary and secondary mirrors to be the greatest gravity-driven optical misalignment. This decentration is expected to have a maximum value of $18 \mu \mathrm{m}$ from nadir pointing to horizon pointing and introduces a net wavefront error at the image of $11 \mathrm{~nm}$ RMS in the form of coma; i.e., negligibly small.

A modal analysis has been performed for the design using ANSYS, for frequencies up to $1000 \mathrm{~Hz}$ (Fig. 12). Although the structure is most susceptible to torsional loads, analysis results indicate that the fundamental modal frequency is in excess of $120 \mathrm{~Hz}$ (Fig. 12). Published

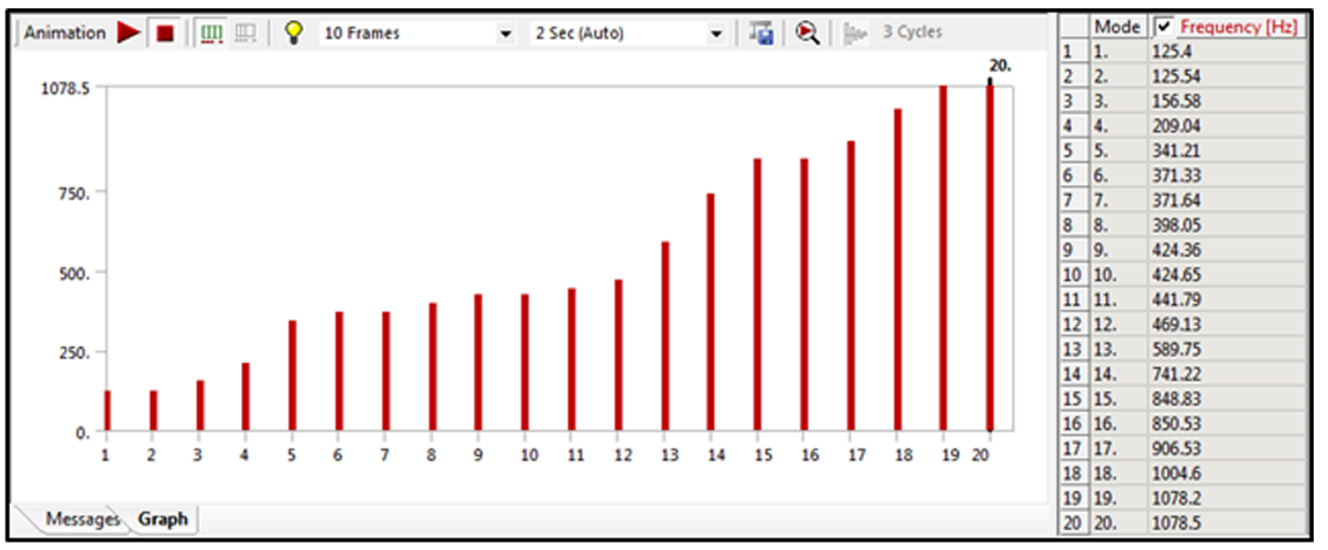

(a)

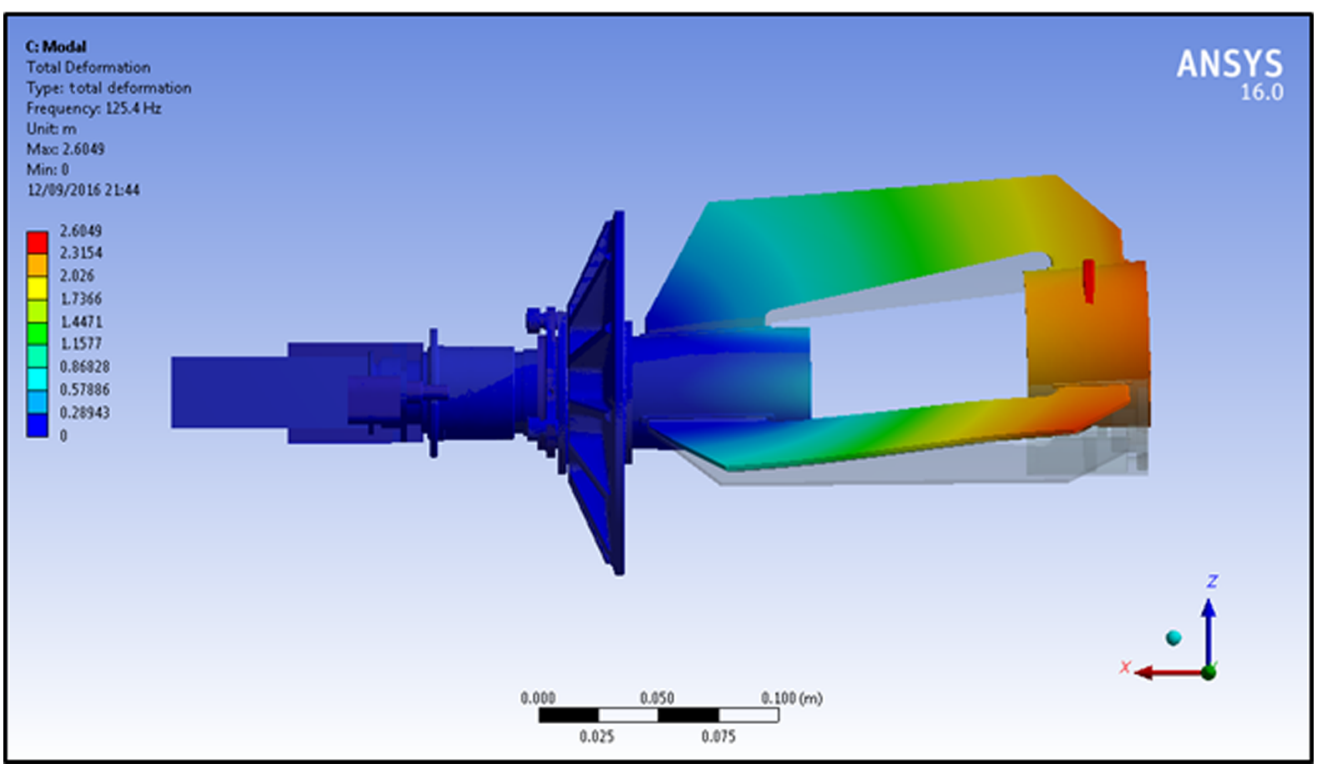

(b)

Fig. 12 Modal analysis results for the camera system: (a) the modal analysis results summary and (b) the deformation behavior associated with the principle vibration mode. 
Table 5 Mass breakdown of the camera system. See the enumerated comments in the text for the scope of each subassembly.

\begin{tabular}{lc}
\hline \hline & Mass $(\mathrm{kg})$ \\
\hline Tube assembly (a) & 0.77 \\
Focus mechanism (b) & 0.52 \\
Drive and supervisory control (c) & 0.35 \\
Gimbal assembly (d) & 0.64 \\
Total & 2.28 \\
\hline \hline
\end{tabular}

vibration data are not currently available for this type of aircraft. However, from published data for composite airframes of similar wingspan, ${ }^{6,7}$ a principle vibrational mode lower than $20 \mathrm{~Hz}$ is expected for the platform. Vibration modes with higher frequencies are reported, but it is unclear if these higher-frequency modes extend beyond $100 \mathrm{~Hz}$. While this suggests that the vibrational modes of the instrument will not be excited by the aircraft, more representative vibration data are being sought.

Table 5 presents the mass breakdown of the camera system, divided into its main subassemblies. In Table 5, the tube assembly, (a), includes the mass of the primary and secondary mirrors and the tube structure. The focus mechanism, (b), includes the corrector lens assembly, the focus assembly, its servo actuation, and the packaged machine vision sensor. Of the $0.52 \mathrm{~kg}$ presented in Table 5 (b), the servo-actuator, external encoder with $2 \mathrm{~m}$ of cable total $0.22 \mathrm{~kg}$. The camera and the focus mechanism require supervisory control, (c). The listed mass is for the COTS desktop arrangement used during testing and includes an embedded micro-PC and associated cabling. For a flight instrument, it is expected that the embedded control computer will be a suitable small-format system with lower mass. The total mass of the camera system is $1.64 \mathrm{~kg}$.

There is a gimbal arrangement included in the design for this prototype camera system, (d). This mass includes the physical interface between the payload and the platform. It is expected that the operational camera system will be enclosed, and that the gimbal arrangement and its actuation will form part of this enclosure assembly. The gimbal mass of $0.64 \mathrm{~kg}$ gives a total mass for the camera system and gimbal of $2.28 \mathrm{~kg}$, which conforms to the requirement of $<3 \mathrm{~kg}$ and leaves a mass "surplus" of $0.72 \mathrm{~kg}$ available for an aerodynamic enclosure for the instrument.

The maximum payload volume is specified as $24,000,000 \mathrm{~mm}^{3}$, with a volume of $150 \times 150 \times 400(=9,000,000) \mathrm{mm}^{3}$ allocated to the camera system. The current design occupies a volume of $150 \times 150 \times 438(=9,855,000) \mathrm{mm}^{3}$. While not conformant with the length specification, this length includes the COTS machine vision camera $(57.5 \mathrm{~mm})$. The length of the instrument is driven by the focal ratio of the primary mirror, the length of the focus mechanism, and the sensor packaging. Some of the excess length can be mitigated by folding the optical beam between the focus mechanism and the sensor assembly. This simple modification has the potential of removing up to $55 \mathrm{~mm}$ from the total length of the instrument, while adding $<0.02 \mathrm{~kg}$ to the total mass from an additional fold mirror.

Table 6 presents power consumption data for the camera system. The total power consumption is expected to be $\sim 28 \mathrm{~W}$ with all motor servos operating. This power consumption is well within the specification of $250 \mathrm{~W}$ peak power.

The expected natural frequency of the instrument is $>100 \mathrm{~Hz}$, which is expected to be higher than any excitation vibration modes produced by the platform in flight. However, measured vibration data for these platforms are not yet in the public domain.

\section{Thermal Design}

There are three aspects to the thermal behavior of the system throughout the specified temperature range: 
Rees et al.: Development of a lightweight camera for high altitude platform systems

Table 6 Power consumption breakdown of the camera system. The data are based upon laboratory measurements.

\begin{tabular}{lc}
\hline \hline Component & Power consumption \\
\hline COTS supervisory PC & 8 \\
Packaged sensor & 5.2 \\
Focus servo assembly & 4.85 \\
Gimbal servos & 9.7 \\
Total & $27.75 \mathrm{~W}$ \\
\hline \hline
\end{tabular}

(1) The structural integrity of the system.

(2) The effects of localized heat sources.

(3) The performance of the optical system.

Because of the diversity of materials used in the camera system, it was necessary to use an epoxy structural adhesive to bond disparate materials in the structure: specifically, the secondary mirror to its support and the CFRP vanes to their aluminum interfaces. 3M Scotch-Weld DP190 adhesive was used for both interfaces. While analysis confirmed the strength of this adhesive for this application, the significant variation in CTE between the adhesive and the structural materials meant that experimental verification of the structural integrity was required during environmental testing of the instrument.

At atmospheric pressures representative of operation at altitude, only radiative cooling is available to remove heat dissipated by local heat sources. As a result, any components that dissipate power must either be packaged to allow effective radiative cooling or have a conductive "bridge" to a correctly specified radiative heat sink. The DC servo motor and camera sensor both dissipate several Watts during operation. However, while the focusing function is designed to be intermittent and can be powered off when not in use, the camera sensor remains powered on throughout. A maximum dissipation of $4.5 \mathrm{~W}$ is documented for the packaged camera sensor and requires a strategy for removing the thermal load from the vicinity of the primary mirror.

Figure 13 shows the effects of the heat dissipation from the camera sensor over the operational range of temperature and pressure, modeled using ANSYS. For high environmental temperatures $\left(+30^{\circ} \mathrm{C}\right)$, the sensor packaging will reach nearly $+52^{\circ} \mathrm{C}$. At altitude, where the specified temperature is $-40^{\circ} \mathrm{C}$, conduction through the titanium structure enables this structure to act as a suitable radiative heatsink for the packaged camera sensor. Here, the sensor packaging reaches a temperature of $+29^{\circ} \mathrm{C}$. This indicates that heating is expected to be localized for an operational sensor choice of similar dissipation, and that modest radiative cooling measures are a tractable approach to removing this dissipated heat from the vicinity of the large optics.

By the careful use of structural materials with differing CTE, the design of the instrument is intended to maintain the optical system near focus throughout its operational temperature range. For the final structural design, the instrument drifts approximately half a wave in focus throughout its specified temperature range (Fig. 14). This defocus behavior was confirmed during environmental testing.

\section{System Performance}

Two prototype instruments have been built and tested. Of these, one has remained unmodified and one has been modified to accommodate the findings of earlier system tests. These instruments have been subjected to both a laboratory testing campaign and environmental testing. 


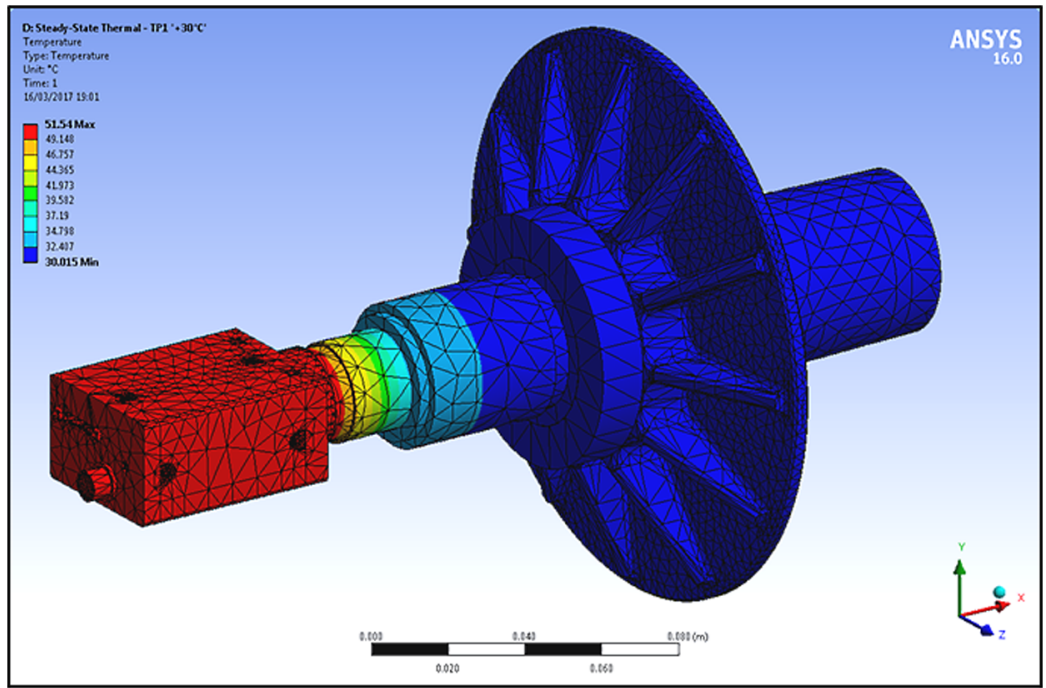

(a)

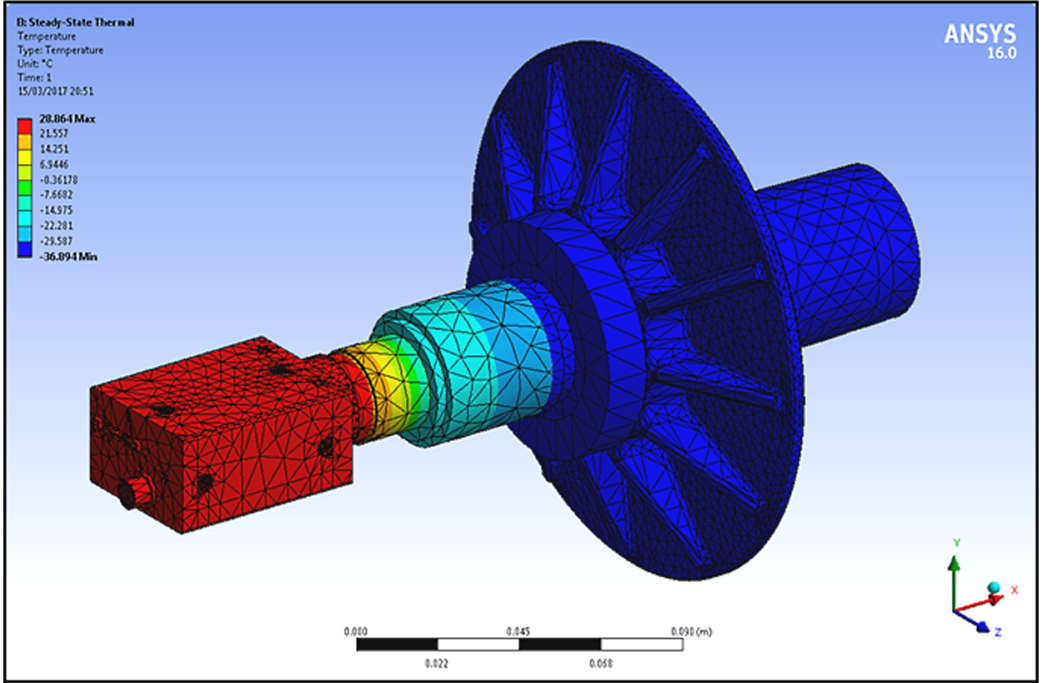

(b)

Fig. 13 FEA of heat flow dissipated from the camera sensor packaging through the focusing mechanism assembly. These data are steady state results. (a) At a temperature of $+30^{\circ} \mathrm{C}$, 1000 mbar pressure the sensor can reach $+52^{\circ} \mathrm{C}$; and (b) at a temperature of $-40^{\circ} \mathrm{C}$ and $40 \mathrm{mbar}$ pressure the sensor can reach $+29^{\circ} \mathrm{C}$.

\subsection{Laboratory Testing}

The primary and secondary mirrors for both instruments were fabricated in-house. The design of the corrector lenses was modified to use COTS lenses of similar prescription and fabricated from Schott N-BK7. While this optical design change degrades the off-axis performance of the instrument, the on-axis performance remains unchanged.

To define the top-level optical performance of the system, the Maréchal criterion ${ }^{10}$ was used: a "well corrected" optical system is one with a phase variance over the pupil of $<0.2$ waves, indicating a Strehl ratio of $>0.80$ and an RMS wavefront of 1/13.4 waves. The Maréchal criterion indicates that the RMS wavefront irregularity emerging from the optical system must be $47.2 \mathrm{~nm}$ or less to achieve a well corrected or "diffraction limited" optical performance, assuming a test wavelength of $633 \mathrm{~nm}$.

Figure 15 shows the fabricated surface error maps for the primary and secondary mirrors, after coating, as derived from interferometric data. Table 7 presents a wavefront error budget for the instrument, taking account of fabricated surface accuracy only; i.e., no accommodation 
Rees et al.: Development of a lightweight camera for high altitude platform systems

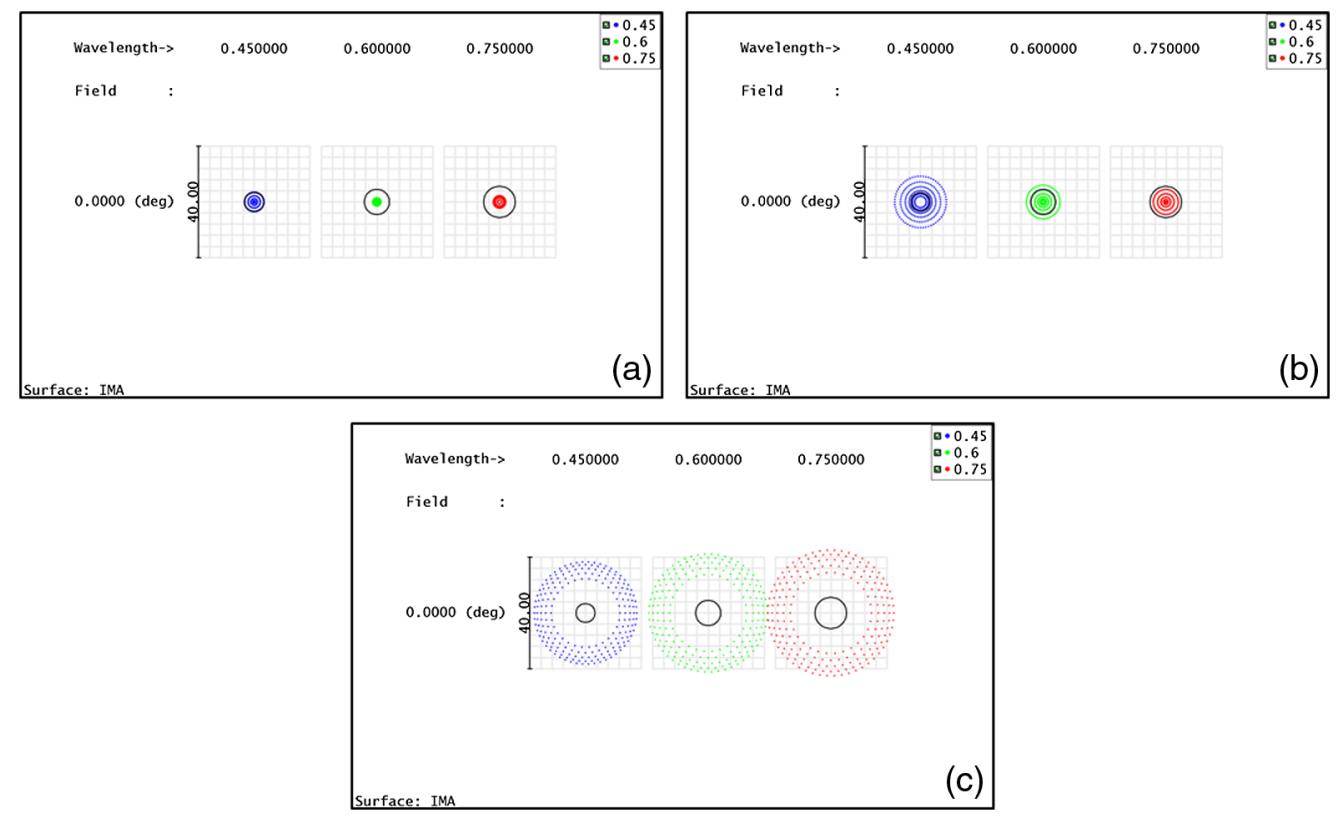

Fig. 14 Spot diagrams resulting from a Zemax thermal analysis illustrating the variation of focus with operational temperature/pressure: (a) $+20^{\circ} \mathrm{C}$, (b) $+30^{\circ} \mathrm{C}$, and (c) $-40^{\circ} \mathrm{C}$.

of sources of wavefront error from integration of the optical system. The COTS lenses were not verified individually, so the manufacturer's quality data were used. Note that while the fullaperture figure error of the COTS lenses is quite large, the optical footprint of the beam is small because of their proximity to the focal plane. This lowers the contribution of figure errors to the propagated wavefront.

It can be seen from Table 7 that the fabricated quality of the optics is sufficient to meet the Maréchal criterion, but with little margin to accommodate the impact of integration upon the system wavefront error. Specifically, we expect wavefront degradation attributable to:

(1) Integration of the optics into their respective cells;

(2) Residual misalignments.

Initial optical alignment of the integrated system was performed using a point source microscope. ${ }^{11}$ Fine optical alignment was performed using interferometric data acquired using a 4D PhaseCam 6000 stabilized Twyman Green interferometer (Fig. 16). In both optical alignment stages, the integrated system was tested in autocollimation against a calibrated optical flat. The expected RMS surface error of the optical flat over the beam footprint was 6-nm; i.e., 12-nm RMS wavefront.

Figure 17 shows the measured wavefront error map of the integrated system after final alignment. The system wavefront error is 52.1-nm RMS and 298-nm PV. This result is to be compared with an expected system wavefront error of 45.1-nm RMS, estimated from the wavefront contribution from each of the optical elements, and a target of 47.2-nm RMS (Maréchal criterion).

The low-order Zernike aberration coefficients derived from these measurements are presented in Table 8. The coma term indicates that optical alignment is sufficient, contributing $15 \mathrm{~nm}$ to the wavefront RMS in root sum square (RSS), but the residual astigmatism is high. The astigmatism contributes $35 \mathrm{~nm}$ to the wavefront RMS in RSS and is the main cause of the wavefront degradation. Further investigation of the astigmatism identified its cause as the deformation of the primary mirror surface from mounting forces and found this effect to be hysteretic.

\subsection{Environmental Testing}

Two sets of environmental tests were conducted on the assembled prototype instruments: those designed to test the survival of the system; and those designed to test the optical performance of the system. 


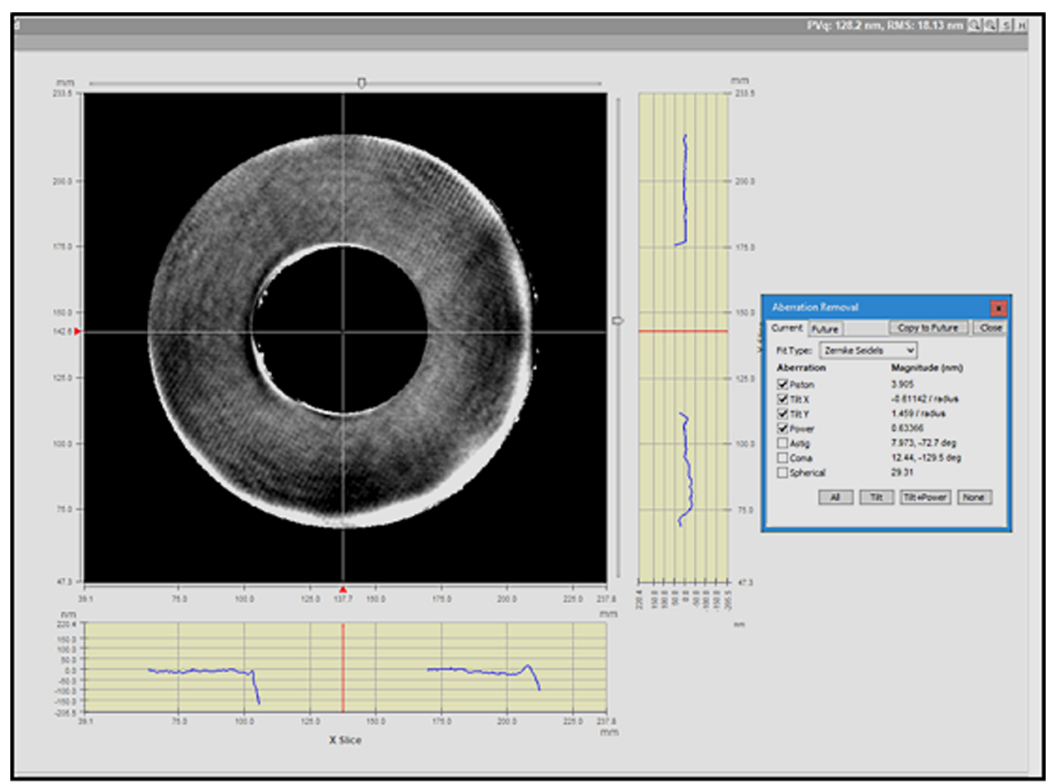

(a)

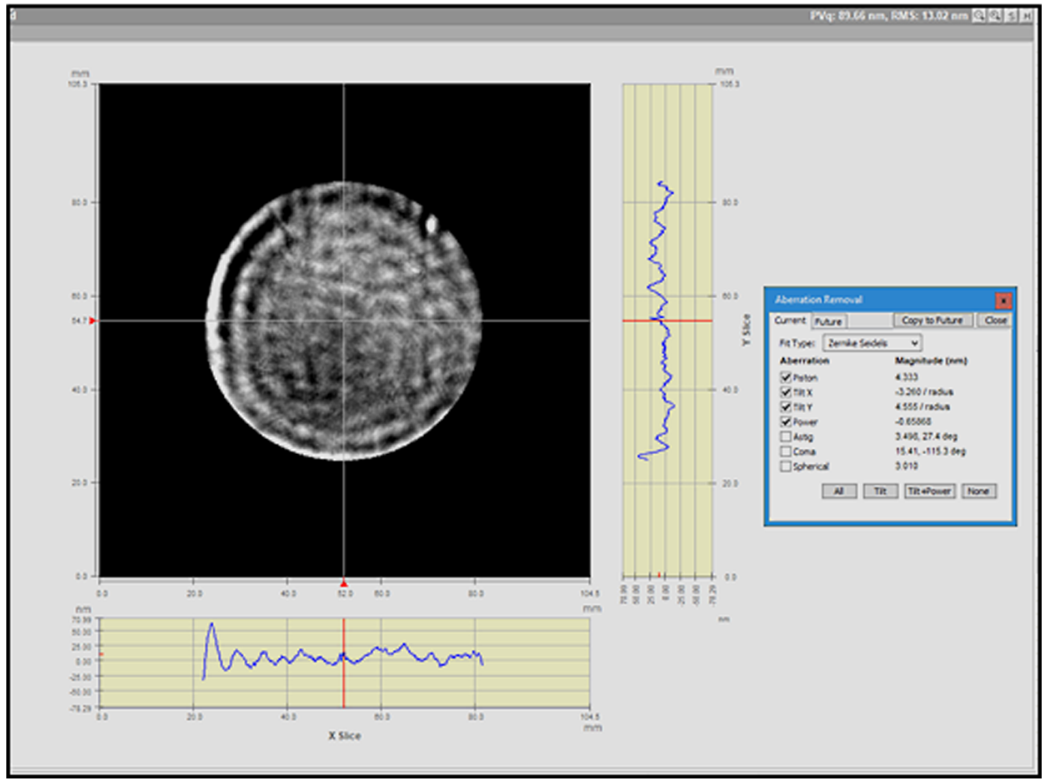

(b)

Fig. 15 Fabricated surface error maps for the primary and secondary mirrors: (a) the primary mirror (18.1-nm RMS surface error) and (b) the secondary mirror (13.0-nm RMS surface error).

Two survival tests were performed:

(1) Survival of the assembly with increased loading at $-80^{\circ} \mathrm{C}$.

(2) Survival of the assembly throughout several $+30^{\circ} \mathrm{C}$ to $-80^{\circ} \mathrm{C}$ duty cycles, designed to replicate a diurnal mission profile.

The tube assembly consists of three CFRP vanes bonded to the aluminum baffles of the primary and secondary mirrors using an epoxy adhesive. To validate the integrity of this subassembly, the unit was loaded to the equivalent of five times gravity and cycled several times from $+30^{\circ} \mathrm{C}$ to $-70^{\circ} \mathrm{C}$ at ambient pressure in an environmental chamber. The duty cycle for the temperature variation was $12 \mathrm{~h}$. Figure 18 shows this loading test. At the end of the test, 
Table 7 Wavefront error budget for each optic. Data for on-axis wavefront performance only. $\mathrm{M} 1$ and M2 are the primary and secondary mirrors, respectively, L1 and L2 are the two corrector lenses.

\begin{tabular}{lcccccc}
\hline \hline Surface & $\begin{array}{c}\text { OCA } \\
(\mathrm{mm})\end{array}$ & $\begin{array}{c}\text { Footprint } \\
(\mathrm{mm})\end{array}$ & $\begin{array}{c}\text { Budgeted full } \\
\text { aperture surface } \\
\text { error (nm RMS) }\end{array}$ & $\begin{array}{c}\text { Budgeted } \\
\text { wavefront error } \\
(\mathrm{nm} \text { RMS) }\end{array}$ & $\begin{array}{c}\text { Fabricated } \\
\text { surface error } \\
(\mathrm{nm} \mathrm{RMS)}\end{array}$ & $\begin{array}{c}\text { Derived } \\
\text { wavefront error } \\
(\mathrm{nm} \text { RMS) }\end{array}$ \\
\hline M1 & 148.6 & 148.6 & 19.0 & 38.0 & 18.1 & 36.2 \\
M2 & 60.0 & 52.6 & 16.9 & 33.8 & 13.0 & 26.0 \\
L1 plano & 22.9 & 8.1 & 63.9 & 97.9 & 97.9 & 6.1 \\
L1 convex & 22.9 & 7.8 & 69.0 & 49.0 & 49.0 & 2.8 \\
L2 concave & 22.9 & 4.7 & 142.4 & 49.0 & 49.0 & 1.0 \\
L2 plano & 22.9 & 4.3 & 170.2 & 97.0 & 97.0 & 1.7 \\
& & & RSS Total & 47.2 & & 45.1 \\
\hline \hline
\end{tabular}

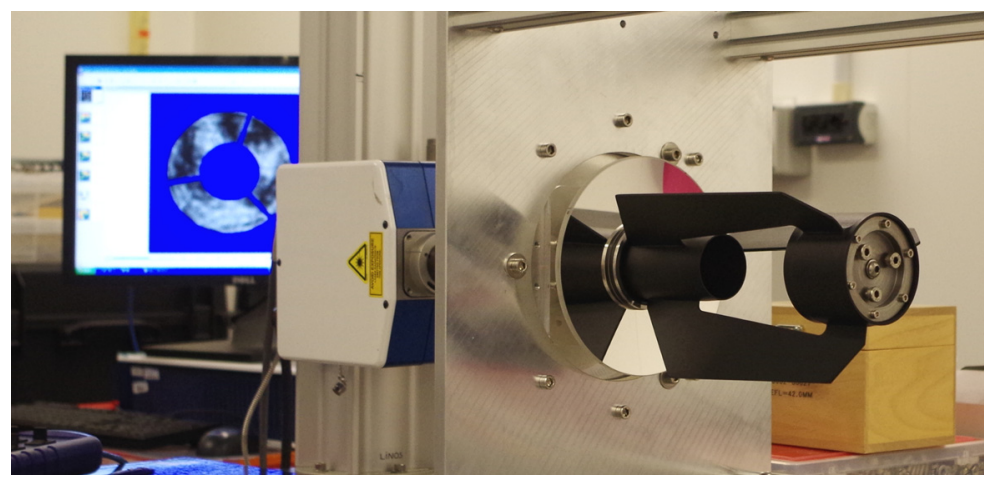

Fig. 16 Interferometric testing of the camera in autocollimation using a 4D Technology PhaseCam 6000 stabilized Twyman-Green interferometer. The return flat is outside the field on the right.

all bonded interfaces were inspected for visible signs of stress fracturing. No visible signs of failure were found under magnification at any of the six interfaces. The integrity of these bonds was monitored throughout the remaining environmental testing program with no visible signs of failure being found.

The camera system was then cycled in the chamber at ambient pressure through the temperature range $+30^{\circ} \mathrm{C}$ to $-70^{\circ} \mathrm{C}$ with the camera sensor and focus actuation enabled. The function of the camera sensor and focus mechanism were checked at ambient and both temperature extremes. Correct function was observed at all test points throughout a 3-day trial.

It is noted that the environmental chamber used for these tests was not a "dry" testing environment. During the test, it was clear that ice was building up on the actuators and mirrors at low temperature. This ice build-up subsequently melted as the unit was cycled through to the higher temperature. However, the unit stayed functional throughout the testing program.

To test the alignment of the optics over the required operating temperature range, a test was devised to use the two imaging systems mounted facing each other (Fig. 19). One system had the COTS machine vision sensor at its focal plane and the other system had a test target at its focal plane (a micromachined plate illuminated by an LED light source). The optical path between these two optical systems was collimated. The camera system was adjusted to provide a focused image of the target.

Once integrated and aligned at room temperature, the test arrangement was placed in an environmental chamber and tested at pressures down to 30 mbar and temperatures down to 


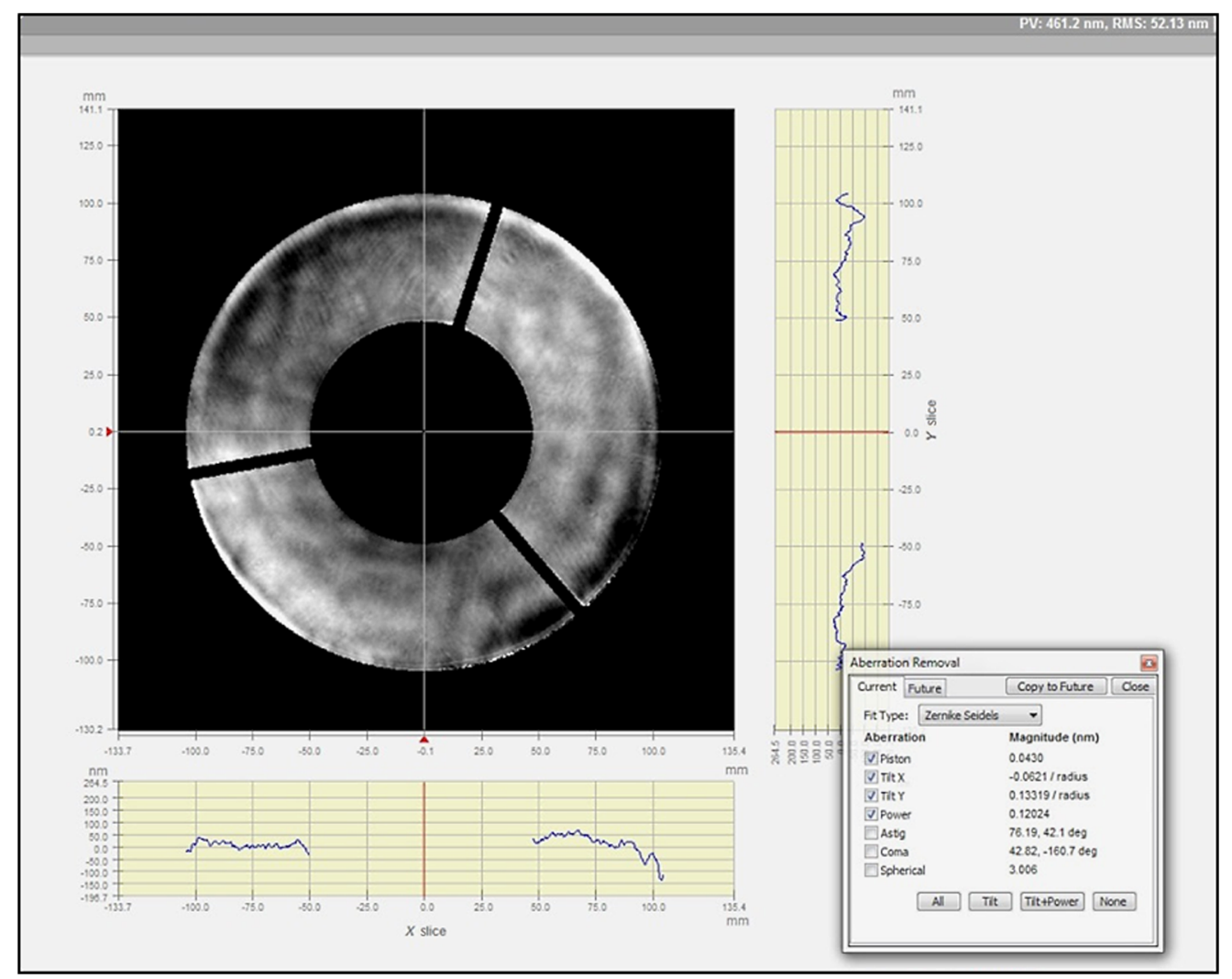

Fig. 17 Final wavefront error map for the optical system. The final measured wavefront error is 52.1-nm RMS and 298-nm PV.

Table 8 Final Zernike terms for the camera system, expressed as wavefront Fringe Zernike coefficients.

\begin{tabular}{lc}
\hline \hline Zernike term & Fringe Zernike coefficient $(\mathrm{nm})$ \\
\hline X-astigmatism & 9.1 \\
Y-astigmatism & 84.9 \\
X-coma & -40.1 \\
Y-coma & -13.8 \\
Primary spherical & +2.5 \\
X-trefoil & -8.4 \\
Y-trefoil & -1.8 \\
\hline \hline
\end{tabular}

$-40^{\circ} \mathrm{C}$, with a test profile designed to represent an expected operational profile. After an initial period of thermalization within the chamber, the test target was focused (Fig. 20). Each illuminated point in Fig. 20(a) is the image of a 15- $\mu$ m diameter pinhole and has a measured full-width at half maximum (FWHM) of $18 \mu \mathrm{m}$. There is some evidence of both flaring and residual coma in these data, which is attributed to a small misalignment in one or each of the two telescope assemblies.

During the test, images of the test target were taken every $10 \mathrm{~s}$. Both defocus and trefoil were apparent in the data at the $-40^{\circ} \mathrm{C}$ test point. The trefoil can clearly be seen in Fig. 20(b); the defocus has been removed. Because the two telescopes are identical and mechanically aligned, 


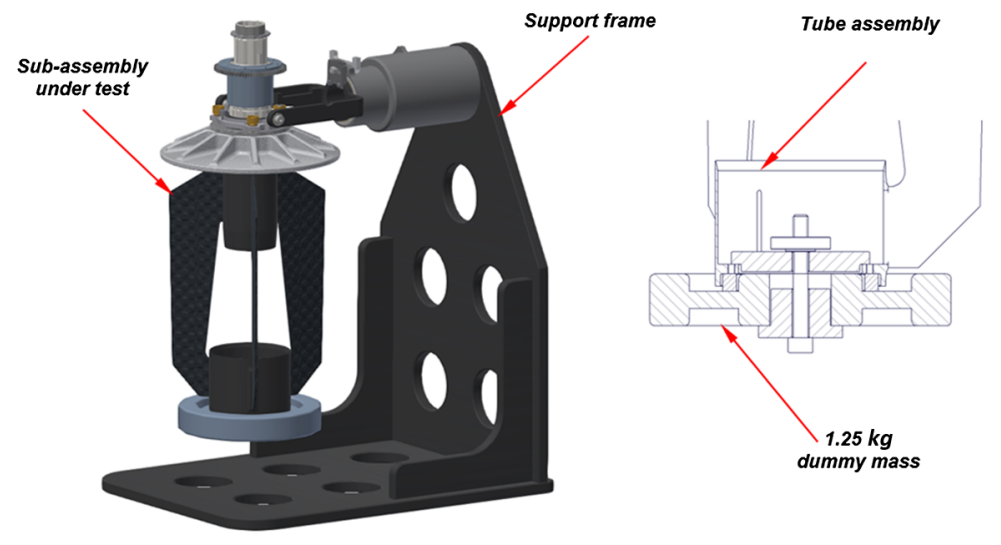

Fig. 18 Assembly for test loading the bonded joints of the instrument.

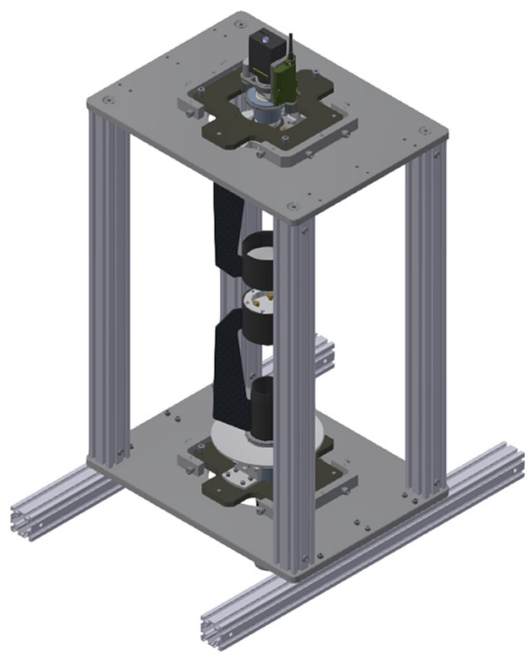

Fig. 19 Test arrangement for environmental verification of optical performance.

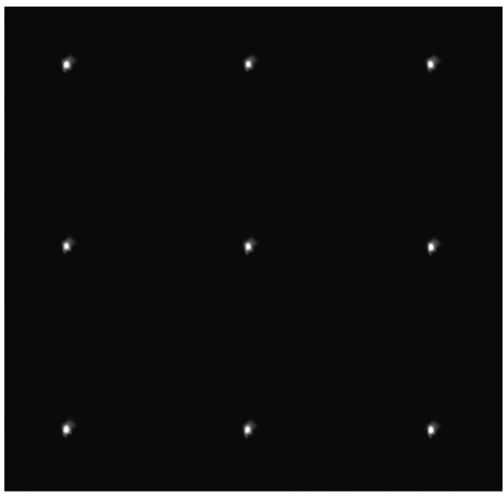

(a)

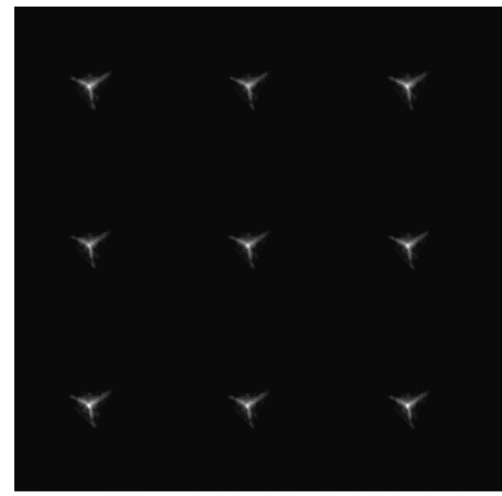

(b)

Fig. 20 Test image data: (a) in focus at the start of cooling and (b) exhibiting trefoil at $-40^{\circ} \mathrm{C}$.

this trefoil must arise in both units. The trefoil is attributed to temperature-induced stress in the secondary mirror caused by its mounting arrangement. It is likely that some of the observed defocus was the result of the same causal factor, but this was not quantified. This mounting arrangement has since been redesigned (see Sec. 8). 


\subsection{Summary}

In summary, the system testing has produced the following results:

(1) From an assessment of the fabrication quality of each of the optical elements, the system wavefront error is expected to be close to that required to deliver the specified optical performance.

(2) The wavefront accuracy of the integrated system, measured under laboratory conditions, diverges slightly from the target wavefront performance.

(3) The cause of the degradation of the expected system wavefront accuracy is variable astigmatism resulting from the influence of the mounting arrangement of the primary mirror.

(4) The key design issue arising from the environmental testing was the temperature-related deformation of the secondary mirror. Investigation has shown this deformation to result from a CTE mismatch between the titanium alloy secondary mirror cell, the N-BK7 mirror substrate, and its rigid interface to an aluminum alloy structural subassembly. The forces induced from this CTE mismatch are transferred between the cell and the optic via the three epoxy bonds between the M2 and its cell.

(5) The variation in focus during environmental testing, while predicted, means that an actuated focus mechanism remains a requirement. This mechanism both increases the weight of the instrument and its complexity. As a result, a passively athermal design is to be sought.

(6) During environmental testing, certain temperature points resulted in significant amounts of icing and condensation on the telescope system. While the telescope system remained functional throughout these tests, further investigation is required of the expected environmental conditions during flight.

Sufficient understanding of the thermal behavior of the system has been acquired to move to a fully passively athermal system design requiring no in-flight focus actuation.

Further environmental testing is required to achieve a status of "flight ready."

\section{Design Outcomes}

On review, we identify several areas where further development would improve the product. These are categorized as follows:

(1) Improvement for optical performance;

(2) Improvement for SWaP conformance;

(3) Reducing production costs.

Three areas of improvement of optical performance are identified:

- Minimizing mounting stresses on the primary mirror;

- Minimizing thermally induced stresses on the secondary mirror;

- Minimizing thermally induced focus variation (athermalization).

Deformation of the primary mirror from mounting stresses results from the "clamping" arrangement between its central bore and the cell. These clamping forces are difficult to assess during integration. They must also remain constant throughout the operating temperature range of the instrument. Replacing this clamping arrangement with a bonded interface using RTV silicone adhesive removes the need for clamping forces in the cell arrangement. The correct selection of RTV silicone adhesive will minimize the risk of thermally induced deformation of the mirror.

For the secondary mirror, design changes for the removal of trefoil aberration seen in the optical performance at low temperature consist of a partial redesign of this subassembly and a change to the cell-mirror interface. The aluminum structural subassembly that interfaces to the titanium alloy cell has been replaced with one fabricated from stainless steel (316) to 


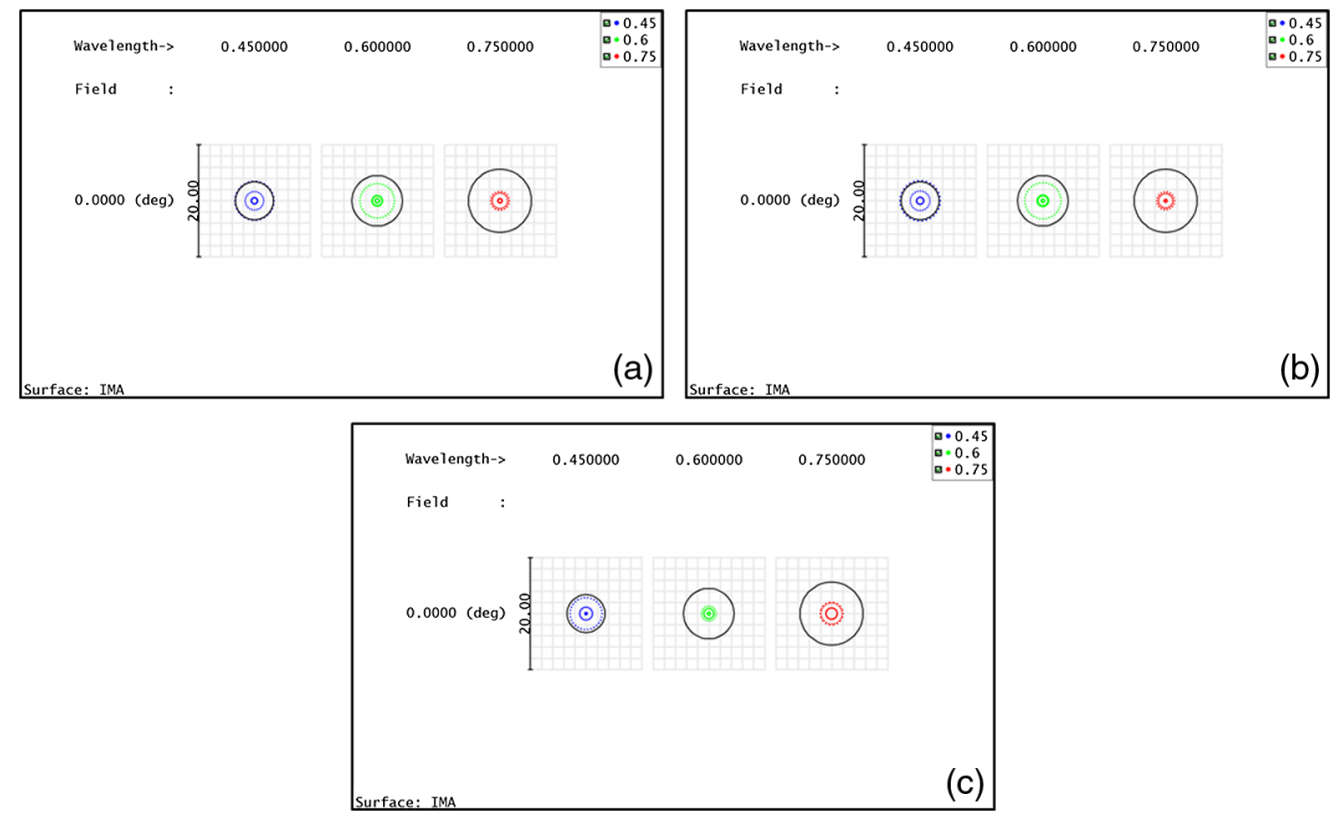

Fig. 21 Spot diagrams illustrating the variation of focus with operational temperature/pressure after changing the primary mirror cell material from aluminum alloy (7075) to stainless steel (314): (a) $+20^{\circ} \mathrm{C}$, (b) $+30^{\circ} \mathrm{C}$, and (c) $-40^{\circ} \mathrm{C}$.

achieve a better CTE match. The three epoxy bonds between the cell and the secondary mirror have been replaced with a single, central bond at the rear of the secondary mirror. A review of the adhesive choice may also be required but awaits the results from further environmental testing.

A review of the temperature-driven focus behavior was performed with a goal of full passive athermalization over the specified operating temperature range. Reanalysis of the temperaturedriven interaction between the optical system and the structure of the instrument indicates that a change in composition of the primary mirror cell from aluminum alloy (7075) to stainless steel (314) brings the optical system to near full passive athermalization. This is shown in Fig. 21 and can be compared directly with the performance of the existing design shown in Fig. 14.

The SWaP performance of the camera system is conformant except for the physical length of the instrument. The nonconformance is smaller than the length of the COTS machine vision sensor packaging and may be resolved by folding the beam between the rear of the primary mirror and the focal plane.

The authors are aware that for HALE operations the smallest practicable payload crosssection can improve both aerodynamic performance of the platform and mission duration. Reducing the size of the instrument also allows greater flexibility when the telescope is used as the fore optics to additional instrumentation within the SWaP volume. By removing the focus mechanism, reducing the focal ratio of the primary mirror to a maximum of $f / 1.7$ and using a fold mirror to illuminate the focal plane (Fig. 22), it is possible to shorten the total length of the instrument to nearer $300 \mathrm{~mm}$. The optical "cost" of this change is the requirement for a threeelement field corrector to achieve similar optical performance, the introduction of a fold mirror to the design (and thereby its impact upon the wavefront) and increased sensitivity to misalignment. Further reduction in primary mirror focal ratio below $f / 1.7$ is feasible but will result in increasing sensitivity to optical misalignment and require greater complexity in the design of the field corrector.

We identify the following production cost drivers for this design:

- Optical manufacturing costs: The manufacturing costs of highly light-weighted aspheric optics of this size are a significant proportion of the total cost of each unit. While the productionization of the manufacturing process will recover some of these costs, it is appropriate to review the light-weighting design and manufacturing method of the primary mirror. 


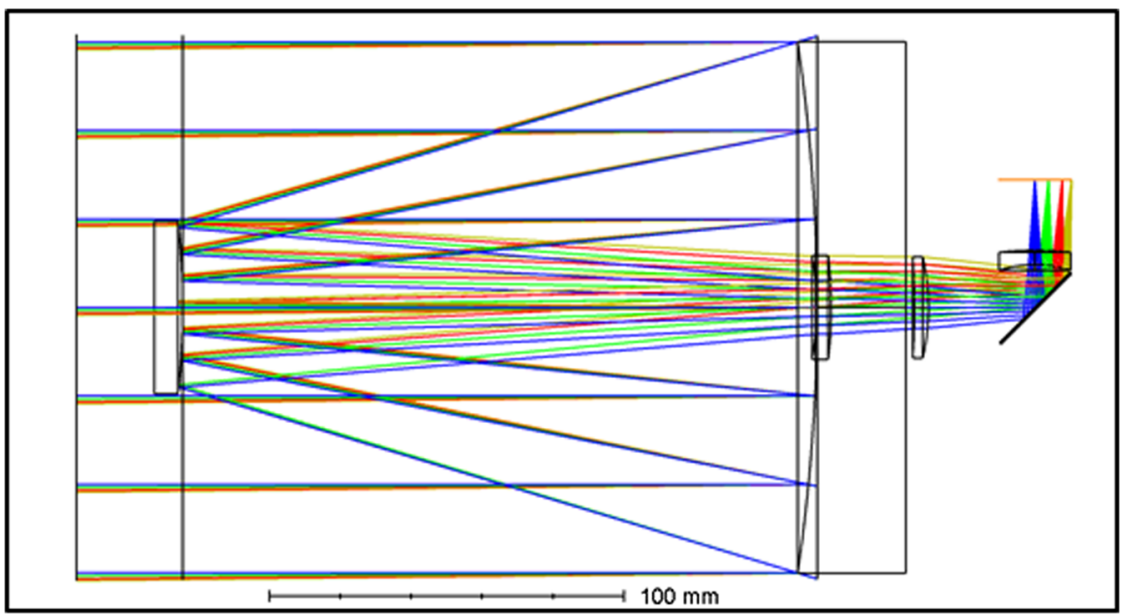

(a)

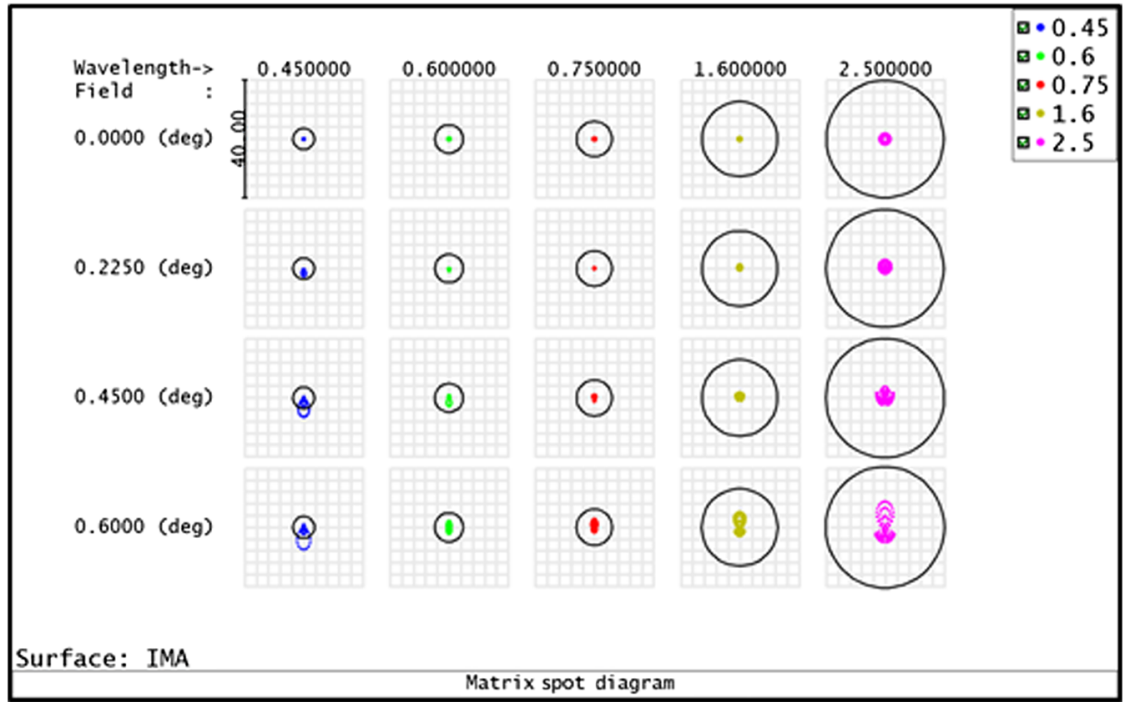

(b)

Fig. 22 (a) Layout of an optical design using an $f / 1.7$ primary mirror with a fold prior to the focal plane and (b) the resulting matrix spot diagram. Note that the optical design does not perform as well as the existing design, despite using a three-element field corrector. This design just fits within $300 \mathrm{~mm}$ instrument length.

- Assembly of the tube structure: The epoxy bonding of structural components incurs a significant labor cost. Once again, the productionization of this process (including improved bonding fixturing) will recover some of these costs. However, other assembly designs may offer a cost advantage over the diversity of materials used here.

- Alignment and interferometric verification of the instrument: Typically, final alignment and interferometric verification in the laboratory takes a minimum of 2 days when starting from a mechanically assembled optical system. Once again, optical alignment as part of a production process requires bespoke alignment jigs to be designed and manufactured. For a system like this, a final alignment and verification program lasting no more than half of one day is a production target.

\section{Conclusions}

While HAPS are still very new, they offer a unique set of aerial capabilities that sit between conventional aircraft and space-borne platforms, particularly in the areas of surveillance and communications. They also have considerable potential for scientific exploitation, offering the 
possibility of access to Earth observation and atmospheric monitoring instrumentation at a lower cost than space-borne experiments and with a more rapid development timescale between concept and deployment.

In this paper, we have reviewed the design and testing of a prototype high-resolution camera suitable for deployment on HAPS as a reconnaissance instrument. In summary, while the emerging HAPs market presents quite specific requirements for optical systems in terms of both SWaP and environmental conditions, a high-resolution camera system is both technically feasible for HAPS deployment and economic to manufacture. We highlight the key SWaP attributes of this system as:

- Total payload mass (without enclosure): $2.3 \mathrm{~kg}$;

- Total payload power consumption: $28 \mathrm{~W}$;

- Total payload volume: $150 \times 150 \times 438 \mathrm{~mm}^{3}$.

An instrument such as the one described has significant potential as a reconnaissance tool, or as the fore-optics to a LIDAR or spectroscopic instrument.

Because of their SWaP and athermal behavior, these technologies are appropriate for deployment on other platforms, from airborne and space-borne to ruggedized instruments for landbased use. We observe the launch vibration requirements to be a design driver for deployment on a space-borne platform. Similarly, land-based operational use requires design effort to protect the optical system from the frequent shock loading likely from operational use.

Inevitably, the use of this design as fore-optics for other instrumentation, e.g., spectroscopic or LIDAR, will lead to design changes aimed at optimizing the system for each role. However, the relative simplicity of the optical design enables modification over quite a wide range of system characteristics without changing the design fundamentals. This is an important feature of the system from the standpoint of its commercial exploitation.

\section{Acknowledgments}

The authors acknowledge the UK Defence Science and Technology Laboratory for its funding of this program and its continued interest in its progress: contract DSTLX1000098365 and contract DSTLX1000103597. We thank Airbus, Farnborough, for the use of their environmental chamber during our testing program. We also thank the Centre for Solar Energy Research, St. Asaph, for the use of their environmental chamber during our testing program. We acknowledge the hard work of the Glyndŵr Innovations production team in fabricating and testing the bespoke optics used in this program. While small, these optics remain challenging to manufacture to the required specification.

\section{References}

1. M. Pent, T. Tozer, and J. Delgado-Penín, "HAPs for telecommunications and surveillance applications," in European Conf. Wireless Technology (ECWT2002) (2002).

2. N. Perlot et al., "System requirements for optical HAP-satellite links," in 6th Int. Symp. Commun. Syst., Networks and Digital Signal Process., Graz, pp. 72-76 (2008).

3. J. Gonzalo et al., "On the capabilities and limitations of high altitude pseudo-satellites," Prog. Aerospace Sci. 98, 37-56 (2018).

4. International Organisation for Standardisation, Standard Atmosphere, ISO 253:1975 (1975).

5. “Airbus Zephyr datasheet," February 2019.

6. J. R. Banerjee, "Modal analysis of sailplane and transport aircraft wings using the dynamic stiffness method," J. Phys.: Conf. Ser. 721, 012005 (2016).

7. J. Simsiriwong and R. W. Sullivan, "Experimental vibration analysis of a composite UAV wing," Mech. Adv. Mater. Struct. 19, 196-206 (2012).

8. L. Jones, "Reflective and catadioptric objectives," in Handbook of Optics, Vol. II, 2nd ed., M. Bass et al., Eds., McGraw-Hill, New York (1995).

9. C. G. Wynne, "Field correctors for large telescopes," Appl. Opt. 4(9), 1185-1192 (1965). 
10. A. Maréchal, "Etude des effets combinés de la diffraction et des aberrations geometriques sur l'image d'un point lumineaux," Rev. Opt. 9, 257-297 (1947).

11. R. E. Parks and W. P. Kuhn, "Optical alignment using the point source microscope," Proc. SPIE 5877, 58770B (2005).

Paul C. T. Rees is a professor of optics: metrology and technology at Wrexham Glyndŵr University, North Wales. He received his BSc degree in astronomy in 1979 and his PhD in astronomy in 1990 both from the University College London. He is a lead systems engineer and project scientist with over 20 years' experience in professional telescope engineering and commissioning. His current interests are the fabrication of large and challenging optics, the metrology of optical surfaces, and the design of airborne optical systems. He has been a fellow of the Royal Astronomical Society since 1984 and is a member of SPIE.

Ian P. Baker is director of Research and Development (Optical Systems) at Gooch and Housego Ltd., where he leads the engineering team at "G\&H St. Asaph" and liaises across the wider G\&H group of companies to provide optical and opto-mechanical expertise. He is a mechanical engineer with 30 years' experience, proven theoretical and analytical expertise and a strong practical background developed through working in and leading multi-disciplinary teams. He has delivered engineering solutions for scientific, medical and defence applications and for operation in harsh environments, high vacuum and space.

David A. Thomson is a highly experienced senior engineer with a broad range of skills in project management, telescopes, optics, and electrical design. He has over 40 years' experience working on professional telescopes worldwide and on major capital projects in the nuclear industry. He is registered with the UK Engineering Council as an Incorporated Engineer and a member of the UK Institute of Engineering and Technology.

Dean Catterall: Biography is not available.

Martin Coleman is principal engineer at Glyndŵr Innovations Limited. He acquired his comprehensive knowledge of precision, opto-mechanical assembly techniques as a mechanical design engineer working for various optical manufacturing companies based in the North Wales opto-electronics cluster. He is responsible for a multidisciplinary engineering team and maintains an active involvement in the day-to-day engineering work, including the development of innovative products and solutions from concept through to delivery.

Martyn Jones is an experienced engineer who completed a master's degree at the University of Liverpool in aerospace engineering and worked in industry as a stress analyst before moving to academia. He is currently a senior lecturer at Wrexham Glyndŵr University, specialising in engineering design and composite materials. He has worked with Glyndwr Innovations Limited as a stress analyst for several years. He recently submitted his $\mathrm{PhD}$ on lightweight CFRP optics.

John B. Mitchell is senior optical metrologist at Glyndŵr Innovations Limited, North Wales. He received his BSc degree in applied physics from University College London in 1987 and his $\mathrm{PhD}$ in optical metrology from Kingston University/National Physical Laboratory in 1996. His current interests are in the precision metrology of large and challenging optical surfaces and in the development of novel optical metrology instrumentation. He has been a member of SPIE since 1992. 